Portland State University

PDXScholar

7-13-1995

\title{
Origins of Effective Charge of Multivalent lons at a Membrane/Water Interface and Distribution of 2,3,4,5-Tetrachlorophenol in a Membrane Model System
}

Piet O. Schmidt

Portland State University

Follow this and additional works at: https://pdxscholar.library.pdx.edu/open_access_etds

Part of the Physics Commons

Let us know how access to this document benefits you.

\section{Recommended Citation}

Schmidt, Piet O., "Origins of Effective Charge of Multivalent Ions at a Membrane/Water Interface and Distribution of 2,3,4,5-Tetrachlorophenol in a Membrane Model System" (1995). Dissertations and Theses. Paper 5049.

https://doi.org/10.15760/etd.6925

This Thesis is brought to you for free and open access. It has been accepted for inclusion in Dissertations and Theses by an authorized administrator of PDXScholar. Please contact us if we can make this document more accessible: pdxscholar@pdx.edu. 


\section{Thesis ApProval}

The abstract and thesis of Piet O. Schmidt for the Master of Science in Physics were presented July 13, 1995, and accepted by the thesis committee and the department.

Committee Approvals:

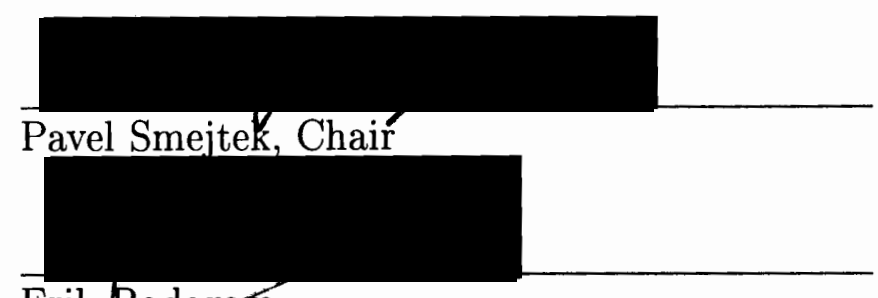

Erik Bodegem

Carl C. Wamser

Representative of the Office of Graduate Studies

DEPARTMENT APPROVAL:

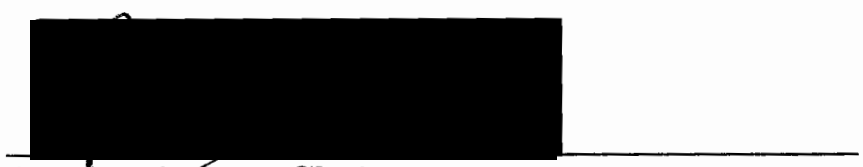

EriklBodegom, Chair

Department of Physics

$* * * * * * * * * * * * * * * * * * * * * * * * * * * * * * * * * * * * * * * * * * * * * * * * * * * * * * * * * * * * * * * * * *$

Accepted for Portland State University by the Library

by

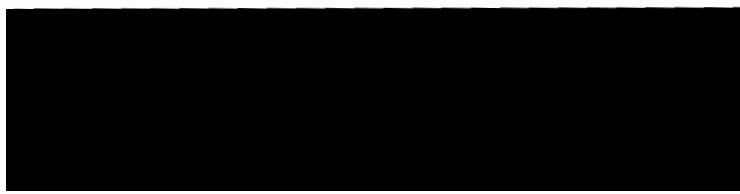

on

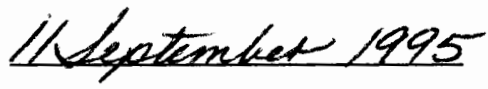




\begin{abstract}
An abstract of the thesis of Piet O. Schmidt for the Master of Science in Physics presented July 13, 1995.

Title: Origins of Effective Charge of Multivalent Ions at a Membrane/Water Interface and Distribution of 2,3,4,5-Tetrachlorophenol in a Membrane Model System
\end{abstract}

Biological cells and subcellular organelles are surrounded by membranes to form compartments performing specialized functions. Adsorption or partitioning of biologically active compounds into the membrane is the first step in the process of modification of cell function. This work is concerned with the problem of distribution of charged molecules between water and electrically charged membrane surface and between water and octanol.

Part I of this thesis is focused on the electrostatic interactions taking place between charges on the membrane and ions present in the aqueous region of the membrane/water interface. The objective was to explore theoretically the origin of anomalous behavior of Ruthenium Red $(\mathrm{RuR})$, a positively charged hexavalent ion. It was discovered in studies of RuR adsorption to negatively charged membranes that within the framework of the Gouy-Chapman theory of the membrane/water 
interface, RuR behaves as an ion with effective charge less than its physical charge. Moreover, the effective charge was found to be dependent on the density of electric charge at the membrane surface. Two theoretical models of the interfacial region were examined: the Rod Model and the Maximum Density Model. The Rod Model takes into account steric constraints imposed on RuR at the vicinity of the membrane surface. The Maximum Density Model attempts to account for non-ideal behavior by including repulsive interactions. These theoretical studies illustrate the consequences of finite size and ion-ion interactions of adsorption of large molecular ions to electrically charged membrane surfaces.

Part II is an experimental study whose objective was to determine the partition coefficient of the negatively charged 2,3,4,5-tetrachlorophenol (TeCP) between water and octanol. The study was based on spectrophotometric measurements of the equilibrium concentrations of $\mathrm{TeCP}$ in water and octanol as a function of $\mathrm{pH}$. The octanol/water partition coefficient for both the non-ionized and ionized species of TeCP were determined. It was found that the partition coefficient of ionized TeCP to lipid membrane is about 400 times greater than that for octanol. This result supports the hypothesis that the octanol/water partition coefficient of ionized chlorophenols cannot be used for predicting their distribution between water and lipid-bilayercontaining elements of the environment. 


\title{
ORIGINS OF EFFECTIVE CHARGE OF MULTIVALENT IONS
} AT A MEMBRANE/WATER INTERFACE

\author{
AND \\ DISTRIBUTION OF 2,3,4,5-TETRACHLOROPHENOL \\ IN A MEMBRANE MODEL SYSTEM \\ By \\ Piet O. Schmidt
}

A thesis submitted in partial fulfillment of the requirements for the degree of

\author{
MASTER OF SCIENCE \\ in \\ PHYSICS
}

Portland State University

1995 
Für

Susi, Ute, Fritz, Jan und Annika 


\section{Acknowledgements}

First and foremost of all I would like to thank my advisor Pavel Smejtek. With his inspiring humor and scientific comprehension he created a productive but at the same time relaxed working atmosphere. Our fruitful discussions helped me to get a better understanding about the "mysteries of physics" with which we were dealing. The experiments would not have been possible without the encouraging help from Shanru Wang. She had the patience to teach me the basic principles of "wet chemistry" and helped me to overcome many obstacles and frustrations. I am indebted to John Dash who supported my interest in electron microscopy and helped me to get the SEM micrograph of a liposome. I enjoyed working with Isolde Sieder and Robert Ward in our lab. Our discussions and late night sessions won't be forgotten! I want to thank the Physics Department here at Portland State for the obligingness I received in various matters and the unbureaucratical way administrative obstacles were overcome. I appreciate the help from Margie Fyfield during the final writeup of the thesis and all the discussions we had throughout the year. I am grateful for the financial support from the Oregon-Baden Württemberg exchange program. I would like to thank all my friends, here and in Germany, who cheered me up during the busiest time of my research. My family had a major influence on my personal and educational development. Without their support I probably would not have been able to be here at Portland State and would have missed this extraordinary experience. Thank you! 


\section{Contents}

Acknowledgements

vi

Introduction to Membrane Systems

I Origins of Effective Charge 5

1 Introduction $\quad 6$

1.1 Overview of Membrane-Electrolyte Models . . . . . . . . . . . . 7

2 Theory 11

2.1 The Gouy-Chapman Model and its Limitations . . . . . . . . . . 11

2.2 The Rod Model . . . . . . . . . . . . . . . . . . . . . . . . 13

2.3 The Maximum Density Model . . . . . . . . . . . . . . . . . . 15

2.4 The Adsorption Model . . . . . . . . . . . . . . . . . . 16

3 Results and Discussion 19

3.1 Electrostatic Calculations . . . . . . . . . . . . . . . . . . 19

3.2 Adsorption Isotherm Calculations . . . . . . . . . . . . . 25

3.3 Conclusions . . . . . . . . . . . . . . . . . . . . . . . 29 
II Distribution of 2,3,4,5-Tetrachlorophenol

4 Introduction

5 Theory 38

5.1 Partitioning Theory . . . . . . . . . . . . . 38

5.2 Distribution Ratio and Partition Coefficients . . . . . . . . . . . . . 42

5.3 Absorption Spectroscopy ... . . . . . . . . . . . . . 43

6 Materials and Methods $\quad 46$

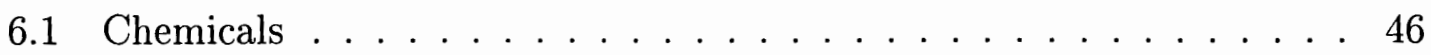

6.2 Spectrophotometric Measurements .............. 46

6.3 Phase Preparation . . . . . . . . . . . . . . 48

6.4 Extinction Coefficients ................. 50

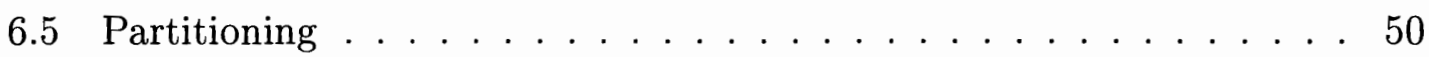

7 Results and Discussion $\quad 52$

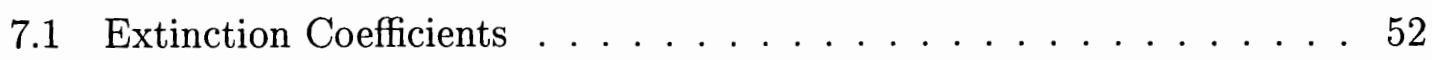

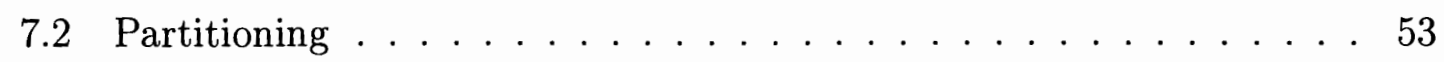

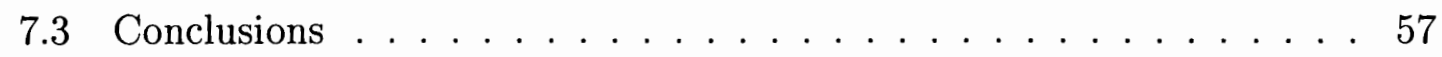

A Derivations $\quad \mathbf{5 9}$

A.1 Gouy-Chapman Theory ... . . . . . . . . . . . 59

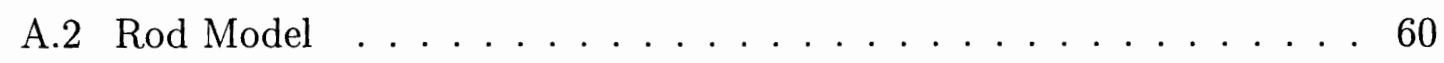

B Numerical Methods $\quad 62$

B.1 Integration . . . . . . . . . . . . . . 63

B.2 Numerical Solution of the GC Model . . . . . . . . . . . . . 63

B.3 Numerical Solution of the Rod Model . . . . . . . . . . . . . . 66

B.4 Numerical Solution of the Maximum Density Model . . . . . . . . . . 68

B.5 Numerical Solution of the Adsorption Model . . . . . . . . . . . 68 
C Spectrophotometer Control Software

Bibliography 


\section{List of Tables}

1 Extinction Coefficients for TeCP . . . . . . . . . . . 53

2 Experimental Data from Partitioning of TeCP . . . . . . . . . . 54

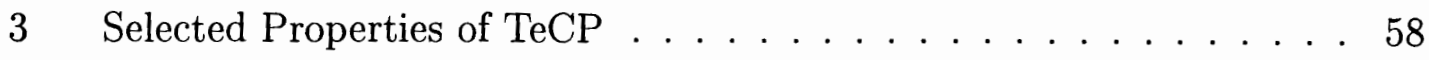

4 List of Parameters stored in a Profile and a Scan File . . . . . . . . 72 


\section{List of Figures}

1 Fluid Mosaic Model of a Membrane . . . . . . . . . . . . . . . . 3

2 Structure of Phosphatidylchline and Model of a Liposome . . . . . . . 3

3 SEM Micrograph of a Liposome . . . . . . . . . . . . . . . . . . . . 4

4 Molecular Structure of 1-Octanol . . . . . . . . . . . . . . . . 4

5 Model Membrane Surface . . . . . . . . . . . . . . . . 8

6 Molecular Structur of Ruthenium Red . . . . . . . . . . . . . . 13

$7 \quad$ Ruthenium Red in front of a Surface . . . . . . . . . . . . . . . 14

8 Potential Distributions for the studied Models . . . . . . . . . . . 20

9 Boltzmann Factors for the studied Models . . . . . . . . . . . . . 21

10 Effective Valency to fit Rod Model . . . . . . . . . . . . . . . 23

11 Effective Valency to fit Maximum Density Model . . . . . . . . . . . 24

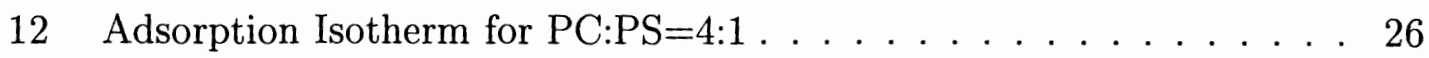

13 Adsorption Isotherm for PC:PS=9:1 . . . . . . . . . . 27

14 Interfacial RuR Concentration . . . . . . . . . . . . . . . 28

15 Primitive Discrete Charge Model . . . . . . . . . . . . . . . 30

16 Molecular Structure of 2,3,4,5-Tetrachlorophenol . . . . . . . . . . 33

17 Reactions at the Octanol/Water Interface . . . . . . . . . . . . 41

18 Energy States in a Molecule and Bathochromic Shift . . . . . . . 44

19 Optical Path in the Spectrophotometer . . . . . . . . . . . . . . 47

20 Absorption Spectra of Presaturated Phases . . . . . . . . . . . . 49

21 Absorption Spectra for TeCP . . . . . . . . . . . . . . 52 
22 Distribution Ratio of TeCP between Octanol and Water . . . . . 55

23 Concentration Profiles of TeCP in Octanol and Water . . . . . . . 56

24 Flowchart for solving the GC Model . . . . . . . . . . . . . . 65

25 Flowchart for solving the Rod Model . . . . . . . . . . . . 67

26 Flowchart for solving the Maximum Density Model . . . . . . . . . 69

27 Flowchart for solving the Adsorption Model . . . . . . . . . . 70

28 User Interface of the Spectrophotometer Control Software . . . . . . 73 


\section{Introduction to Membrane}

\section{Systems}

Processes at membrane surfaces are essential for biological life. Interactions of molecules with the inner and outer surface of cells and organelles trigger a large number of reactions. Adsorption of molecules to membranes is one of the major processes taking place at the membrane/water interface. Information processing in cells is carried out by messenger molecules binding on the outside of the cell and opening an ion channel or triggering the release of a second messenger into the cytoplasma of the cell. For example the hormone epinephrine binds to its receptor on the outside of liver cells triggering a series of reactions inside the cell which ultimately cause the release of glucose (Linder and Gilman, 1992). Another example is the binding of antibodies to foreign cells or to viruses which marks the beginning of the immune system's response, one of the most complex and not yet fully understood systems in higher lifeforms.

Another important function of membranes is to maintain certain ion concentration profiles across the membrane. Nerve cells, for example, maintain a potassium/sodium gradient across their membrane which gives rise to a resting potential of about $-60 \mathrm{mV}$. When an electric action potential propagates along the nerve cell, potassium and sodium channels embedded in the membrane open in sequence and reverse the potential to about $+30 \mathrm{~m} V$ for a time period of one millisecond. The 
respiration chain in mitochondria establishes, via electron transport, a proton gradient across the inner mitochondrial membrane. This results in an electric potential difference which is one of the components of the driving force for the synthesis of adenosine triphosphate (ATP), the major energy source of cells (Stryer, 1981).

Greater knowledge about the processes at membrane/water interfaces aids researchers in medicine and health science to design and develop drugs.

It is the purpose of this study to get a better understanding of adsorption to membranes. The approach is twofold:

1. In Part I, the electrostatic properties of the membrane/water interface are discussed. The main interest here lies in the distribution of highly charged, large molecules in front of a charged bilayer surface. Ruthenium Red (RuR) will be considered as an example of such a molecule.

2. Part II elucidates the difference between membrane adsorption of electrically neutral and charged hydrophobic molecules, and the distribution of these species in a widely used membrane model system consisting of two immiscible phases, octanol and water.

Cells themselves are very complicated objects to study because of their inherent complexity and multifunctionality. Singer and Nicolson (1972) proposed a fluid mosaic model in which membranes consist of a ca. $70 \AA$ thick two-dimensional solution of oriented globular proteins and lipids as indicated in Figure 1. These proteins, which are embedded into the membrane (integral proteins) or adsorbed to it (peripheral proteins), alter the electrochemical properties of the lipid bilayer and make a cell a complex system with many unknown properties.

To apply the laws of physics one has to go back to a simpler system and translate the results to the more complex biological world. Artificial liposomes consisting of charged and uncharged lipids (e.g., phosphatidylcholine or phosphatidylserine) are widely used as a practical and successful model. Figures $2 \mathrm{~b}$ and 3 show the structure of artificial liposomes. Lipids themselves consist of a hydrophilic headgroup pointing 


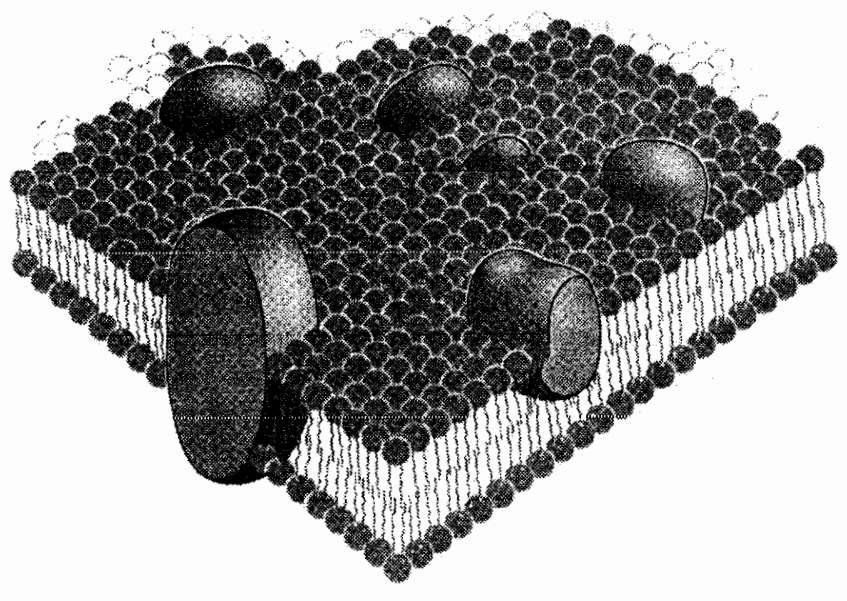

Figure 1: Fluid mosaic model of a membrane according to Singer and Nicolson (1972).

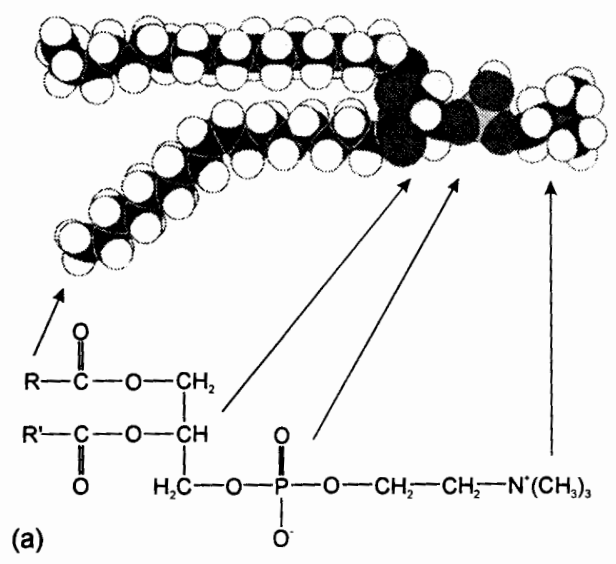

(b)

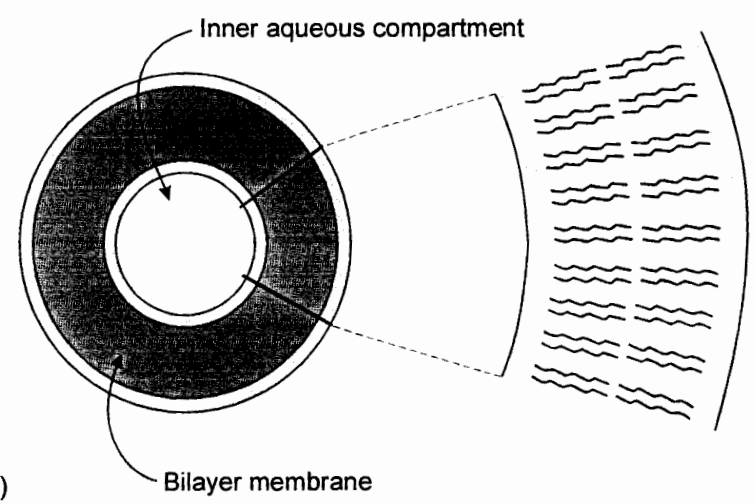

Figure 2: (a) Molecular structure and model of phosphatidylcholine; (b) Structure of a liposome.

to the aqueous phase and a hydrocarbon chain which forms the interior of the bilayer (see Figure 2a).

Another system extensively used as a membrane model is octanol. Octanol has a structure comparable to lipids: a hydrophobic tail (the hydrocarbon part) and a hydrophilic head (the $\mathrm{OH}$ group) as shown in Figure 4. It therefore exhibits similar properties as lipid material including the formation of micelles in aqueous solutions. These properties make octanol to a very successful model system for 


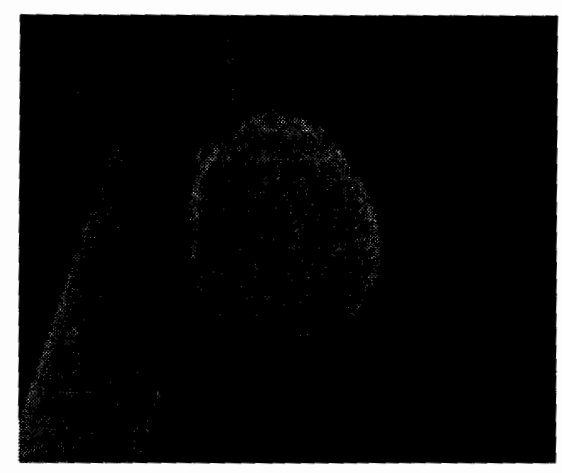

Figure 3: SEM micrograph of an egg-phosphatidylcholine liposome prepared according to Blochel (1992), after fixation with osmium tetroxide and critical point drying (Schmidt, 1995).

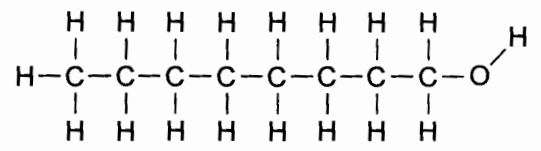

Figure 4: Molecular structure of 1-octanol.

organic matter. Nonetheless it is neccessary to determine the limitations of applying the octanol/water model. 
Part I

\section{Origins of Effective Charge of Multivalent Ions at a Membrane/Water Interface}




\section{Chapter 1}

\section{Introduction}

Adsorption of molecules to membranes is one of the major processes taking place at the membrane/water interface. The density of adsorbed ions is proportional to their interfacial concentration which is given by a Boltzmann relation $N_{i f}=$ $N(\infty) \exp \left(-\frac{z e \psi(0)}{k T}\right)$, where $\psi(0)$ is the surface potential of the membrane and $N(\infty)$ the ion concentration in the solution. Therefore, it is of great importance to study the potential distribution at the membrane/water interface.

The most commonly used model to describe potential distribution at a membrane/electrolyte interface is the Gouy-Chapman (GC) theory. It is the objective of this work to elucidate the role of different approximations made in this approach using data on adsorption of Ruthenium Red (RuR) to artifical liposomes obtained in previous work in this lab (Völker and Smejtek, 1995). It was shown that a satisfactory fit of the experimental data to a Langmuir adsorption isotherm could only be obtained if an effective valency $z_{\text {eff }}<6$ for the hexavalent RuR was assumed. Different surface charge densities were obtained by varying the ratio of charged/uncharged lipids. This revealed that the effective valency approaches its physical value of 6 with decreasing surface charge density.

The need to introduce an effective charge has been reported previously in studies of adsorption of melittin (Schoch and Sargent, 1980; Schwarz and Beschiaschvili, 
1989; Beschiavili and Seelig, 1990) and peptides (Montich et al., 1993; Mosior and McLaughlin, 1992). It is believed that this behavior is due to one or more limitations of the GC theory. To investigate these limitations, numerical calculations of the potential and of the ion distributions have been performed (i) neglecting ion-surface interactions (no adsorption), and (ii) taking into account adsorption in terms of the Langmuir adsorption isotherm. The results were compared with experimental data for RuR adsorption.

\subsection{Overview of Membrane-Electrolyte Models}

There are numerous current models dealing with electrostatic properties of membraneelectrolyte systems. The Gouy-Chapman (GC) theory (Gouy, 1910; Chapman, 1913) of the diffuse double layer is the most frequently used approach to describe those systems. It will be discussed in more detail in Section 2.1. The model treats the surface as infinite, smooth and uniformly charged (see Figure 5). Discreteness of charges ${ }^{1}$ dipole potentials arising from the structure of the lipids, mobility of charges on the surface as well as electrolyte-membrane interactions are neglected. The electrolyte is treated as being ideal ${ }^{2}$ and uniform, ${ }^{3}$ which is the so called primitive electrolyte model. Distributed charge effects and other refinements like molecular roughness of the surface, non-coplanar charge, dielectric constant of the headgroup (Peitzsch et al., 1995) or inclusion of finite membrane thickness (Duniec and Thorne, 1983), have been shown to be important only in special cases not considered here.

Despite the simplicity of the model it can claim remarkable success in predicting experimental results in a variety of systems. ${ }^{4}$ Nevertheless, in certain cases the deviation from experimental results is considerably high. Different approaches have

\footnotetext{
${ }^{1}$ the charge of one lipid occupies about $0.7 \mathrm{~nm}^{2}$ area of membrane surface

${ }^{2}$ ions as charged pointlike noninteracting particles

${ }^{3}$ homogeneous solvent $\left(\epsilon_{r}\right.$ assumed to be constant throughout the aqueous phase), no hydration/solvation effects

${ }^{4}$ for references, see Torrie (1979) and the excellent review paper of McLaughlin (1977)
} 


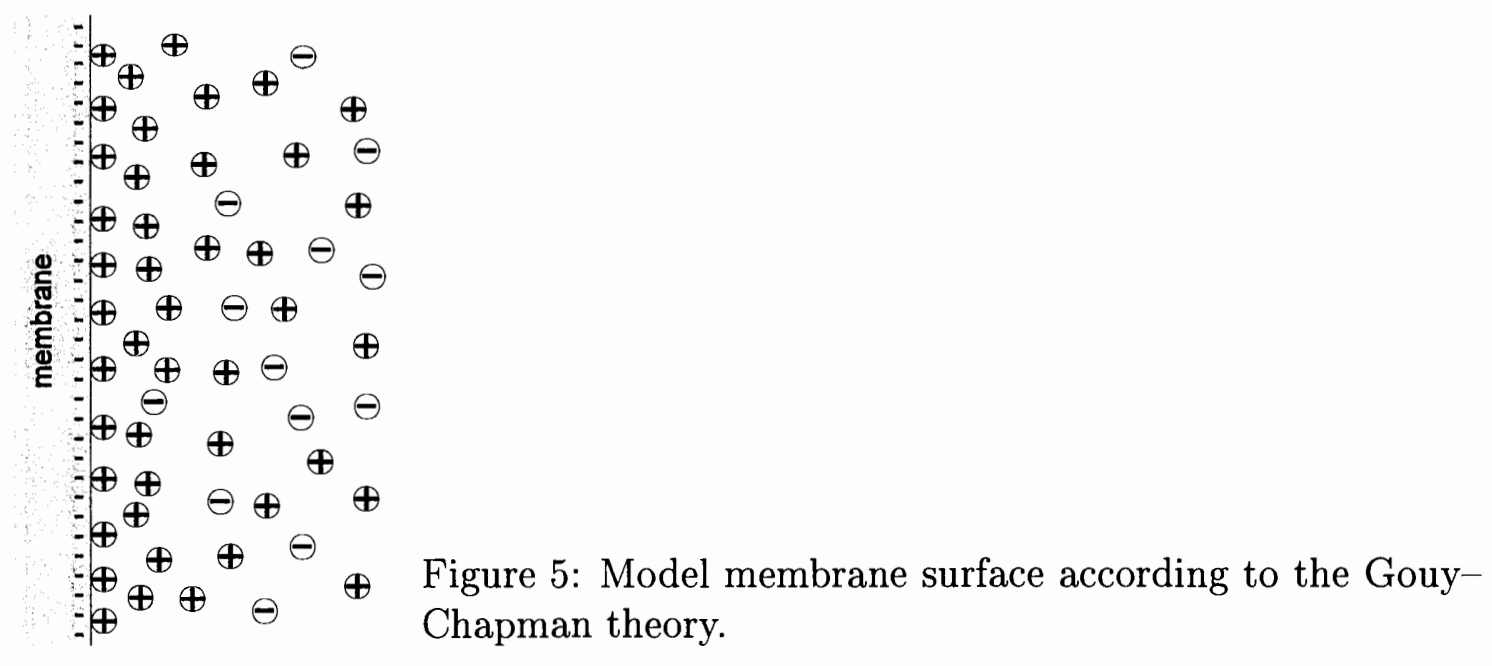

been used to overcome the limitations of the model.

Nelson and McQuarrie (1975) introduced a discrete charge model in which the surface charges form a square lattice of fixed point charges. This model predicts deviations from the smeared charge theory that are larger for counterions than for co-ions. Winiski et al. (1986) performed an experimental test of the model using mono- and divalent ions which agreed much more closely with the GC theory rather than with Nelson's and McQuarrie's model. Discreteness of charge effects are believed to become important with increasing valency of ions (Schoch and Sargent, 1980), adsorption of hydrophilic ions (Andersen et al., 1978) and for the case that the charges are buried into the membrane (McLaughlin, 1977).

Tsien and Hladky (1982) introduced a model based on virial expansion for the electrochemical potential of ions adsorbed into the membrane including ionion repulsion which could satisfactorily explain the findings of Andersen (1978). Stankowski (1991) used a similar approach to describe adsorption of finite size ions of large valency to lipid bilayer. With his model he could explain the effective valency of ions introduced in previous treatments (Schwarz and Beschiaschvili, 1989; Kuchinka and Seelig, 1989; Beschiavili and Seelig, 1990; Stankowski and Schwarz, 
1990) in which the ordinary GC Model was used to fit experimental data to adsorption isotherms.

Another approach is a modification of the Poisson-Boltzmann equation (MPB) in which effects due to local ion densities are taken into account. In this framework the potential is described by a infinite set of potential equations which is truncated by a closure condition of $n^{\text {th }}$-order adding so called "fluctuation potentials" to the potential in the exponential of the Poisson-Boltzmann equation (Outhwaite, 1974).

Very sophisticated models like the Hypernetted Chain approximation ${ }^{5}$ (HNC) for bulk electrolytes which use a statistical mechanics approach on the basis of integral equations including finite size effects (hard spheres) and ion-ion correlations were applied successfully to double layer systems (Carnie et al., 1981; Kjellander and Marčelja, 1985; Kjellander and Marčelja, 1988a; Kjellander and Marčelja, 1988b). Numerical solutions of this and the MPB Model predict damped oscillations (charge inversion) of the potential (Henderson et al., 1979; Bhuiyan and Outhwaite, 1979). Langner et al. (1990) found excellent agreement of experimentally obtained potential distributions using mono-- and trivalently charged lipids with the predictions of HNC.

Monte Carlo methods (MC) provide a means to verify models on a numerical basis. By simulating the diffuse double layer including the main features of the model of interest, one obtains numerical results which then can be compared to the predictions of the model calculations. Due to this totally different approach, it is more of a substitute for experimental work rather than a physical model. One has to remark that this method simulates only the behavior of the double layer within the assumptions of the respective model, rather than the "real world". Torrie and Valleau (1979) showed that the method is applicable to the diffuse double layer problem by testing the predictions of the GC theory using conditions within which the theory is known to work correctly. Further studies (van Megen and Snook, 1980; Torrie and Valleau, 1980) applying grand canonical ensemble Monte Carlo (GCEMC) simulations

\footnotetext{
${ }^{5}$ which is a closure condition for the Ornstein-Zernike (Klein, 1994) integral equation
} 
including finite size and excluded volume effects to high electrolyte concentrations and surface charge densities showed deviations from GC. Torrie and Valleau (1980) compared their results with predictions from GC, MPB and HNC and found that up to electrolyte concentrations of $0.1 \mathrm{M}$ and substantial surface charge densities, GC agrees well with the simulation but HNC and MPB extend their applicability to moderate concentrations and surface charge densities. MC simulations showed that image charges have a significant effect on the ion distribution in front of the surface at low surface charge densities which were well predicted by MPB and, within the framework of the screened self-image, by HNC (Torrie et al., 1982). 


\section{Chapter 2}

\section{Theory}

This chapter introduces the models and their modifications which have been studied. The goal of this investigation was to keep the treatment as physically simple as possible, using the GC theory of the diffuse double layer as the basis of all models. The GC Model has been modified to account (i) for steric constraints imposed on large ${ }^{1}$ ions in front of a membrane surface and (ii) for the finite size of ions which leads to a "volume-pressure" in regions of high ion concentration. The characteristics of these models have been studied in a simple system without ion-membrane interactions to extract the main features and have then been applied to a more realistic model which includes adsorption of ions to the surface according to a Langmuir adsorption isotherm.

\subsection{The Gouy-Chapman Model and its Limita- tions}

The GC theory is based on two fundamental equations: the Boltzmann distribution and the Poisson equation. For ion species $i$ with valency $z_{i}$ at a distance $x$ from the

\footnotetext{
${ }^{1}$ compared to a Debye length
} 
surface, Boltzmann predicts a concentration of

$$
N_{i}(x)=N_{i}(\infty) \exp \left(-\frac{z_{i} e \psi(x)}{k T}\right)
$$

where $N_{i}(\infty)$ denotes the bulk concentration, $k$ is the Boltzmann constant, $T$ the temperature, $\psi(x)$ the potential and $e$ the elementary charge. Poisson's equation is

$$
\frac{d^{2} \psi(x)}{d x^{2}}=-\frac{\rho(x)}{\epsilon_{r} \epsilon_{0}},
$$

where $\epsilon_{r}$ is the uniform dielectric constant of the solution, $\epsilon_{0}$ the permittivity of free space and $\rho(x)$ the volume charge density of the electrolyte. Combining these equations and applying the electroneutrality condition

$$
\sigma_{m}=-\int_{0}^{\infty} \rho(x) d x .
$$

yields the Gouy-Chapman equation ${ }^{2}$ for the diffuse double layer

$$
\sigma_{m}=2 \epsilon_{r} \epsilon_{0} k T \sum_{i} N_{i}(\infty)\left[\exp \left(-\frac{z_{i} e \psi(0)}{k T}\right)-1\right]^{\frac{1}{2}} .
$$

Eqs. 3 and 4 have to be solved numerically. ${ }^{3}$ In many applications this equation is even further simplified by assuming a 1:1 electrolyte and small surface potentials ${ }^{4}$ which yields an analytical solution for $\psi(x)$ (Aveyard and Haydon, 1973):

$$
\psi(x)=\psi(0) e^{-\kappa x} \quad \text { with } \quad \psi(0)=\frac{\sigma_{m}}{\epsilon_{r} \epsilon_{0} \kappa}
$$

where $\kappa$ is the inverse Debye length

$$
\kappa=\left(\frac{2 z^{2} e^{2} N(\infty)}{\epsilon_{r} \epsilon_{0} k T}\right)^{\frac{1}{2}} .
$$

Stern (1924) proposed a correction for the finite size of ions which results in a plane of closest approach ${ }^{5}$ at one effective ion radius, ${ }^{6} r$, away from the surface.

\footnotetext{
${ }^{2}$ for a more detailed derivation see Appendix A.1

${ }^{3}$ see Appendix B.2

${ }^{4} \psi(0) \ll k T$

5 the "outer Helmholtz plane" according to Grahame (Grahame, 1947)

${ }^{6}$ including hydration shell
} 


$$
\left[\left(\mathrm{NH}_{3}\right)_{5} \mathrm{Ru}^{\mathrm{III}}-\mathrm{O}-\mathrm{Ru}^{\mathrm{IV}}\left(\mathrm{NH}_{3}\right)_{4}-\mathrm{O}-\mathrm{Ru}{ }^{\mathrm{III}}\left(\mathrm{NH}_{3}\right)_{5}\right]^{+6}
$$

Figure 6: Molecular structur of Ruthenium Red

The so modified Gouy-Chapman-Stern Model exhibits a linear potential rise in the region $0 \leq x \leq r$ which is equal to $\Delta \psi=\frac{\sigma_{m}}{\epsilon_{r} \epsilon_{0}} r$. This correction does not apply in our case because the potential and the ion concentrations at the outer Helmholtz plane are the same as the ones at the surface without Stern's correction.

The GC Model is very successful in predicting potentials and (together with e.g., a Langmuir adsorption isotherm) adsorption of mono- and divalent ions to bilayer consisting of mono- and divalently charged lipids (Winiski et al., 1986). ${ }^{7}$ However, the model is expected to give false results when dealing with ions large compared to the Debye length or ions with valency greater than two, especially at low surface charge densities.

\subsection{The Rod Model}

Multiply charged molecules whose dimensions are comparable to a Debye length experience steric constraints in front of a charged surface as not all orientations of the molecule are possible. Some orientations are excluded due to the proximity of the molecule to the membrane. In addition, the charge is distributed over the molecule and the molecular ion cannot be regarded to be a pointlike particle. This leads to different potential energies of the charges for different regions of the molecule as they experience different potentials. To include these effects Carnie and McLaughlin (1983) proposed a model with 2 charges on a rigid rod in which the local number density of each $+2 e$-charge on the rod is calculated separately averaging over the possible positions of the other charges. It was neccessary to expand this approach for RuR (see Figure 6), which is modeled as an infinitesimal thin rod of length $a=1.2$ $\mathrm{nm}$ (Carrondo et al., 1980) to include three $+2 \mathrm{e}$ charges A,C at the ends and B, in

\footnotetext{
${ }^{7}$ see e.g. McLaughlin (1977) for a detailed review
} 


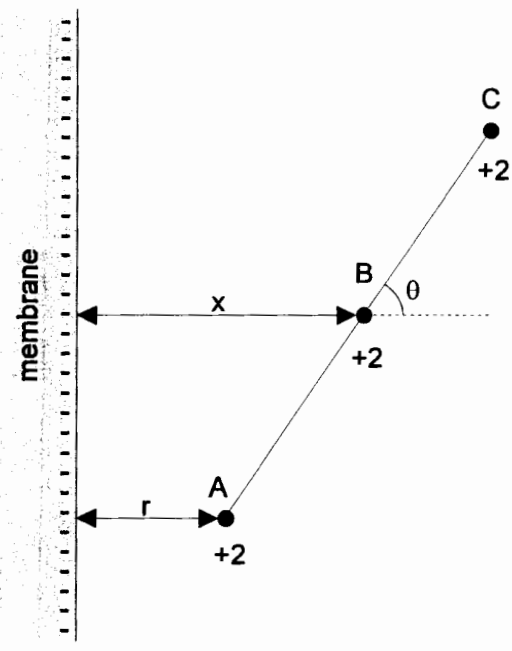

Figure 7: Ruthenium Red Rod-Model in front of a charged surface

its center as shown in Figure 7).

Following the arguments of Carnie and McLaughlin (1983), the local density of charges type $\mathrm{A}$ is given by

$$
N_{A}(r)=N_{A}(\infty) \exp \left(-\frac{2 e \psi(r)}{k T}\right)\left\langle\exp \left(\frac{2 e}{k T}[\psi(r+z / 2)+\psi(r+z)]\right)\right\rangle,
$$

where $\langle\cdots\rangle$ denotes the spatial average over all positions which the other two charges can occupy. Applying the symmetry of the molecule, we obtain $N_{A}(r)=$ $N_{C}(r)$.

Similarily, for the density of charges type B we obtain

$$
N_{B}(x)=N_{B}(\infty) \exp \left(-\frac{2 e \psi(x)}{k T}\right)\left\langle\exp \left(-\frac{2 e}{k T}[\psi(x-z / 2)+\psi(x+z / 2)]\right)\right\rangle .
$$

Expressions for the spatial averages are derived in Appendix A.2. These are

$$
\begin{aligned}
& N_{A}(r)=N_{A}(\infty) e^{-\frac{2 e \psi(r)}{k T}} \frac{1}{2 a} \int_{\max [-a,-r]}^{a} d z e^{-\frac{2 e}{k T}[\psi(r+z / 2)+\psi(r+z)]} \\
& N_{B}(x)=N_{B}(\infty) e^{-\frac{2 e \psi(x)}{k T}} \frac{1}{2 a} \int_{\max [-a,-2 x]}^{\min [a, 2 x]} d z e^{-\frac{2 e}{k T}[\psi(x-z / 2)+\psi(x+z / 2)]} .
\end{aligned}
$$


These densities together with the Poisson equation, Eq. 2, yield, after formal integration,

$$
\psi(x)=\psi(0)+\left.x \frac{d \psi(x)}{d x}\right|_{x=0}-\frac{1}{\epsilon_{r} \epsilon_{0}} \int_{0}^{x} d y(x-y) \sum_{i} z_{i} e N_{i}(y)
$$

This equation has to be solved numerically ${ }^{8}$ using the electroneutrality condition of the diffuse double layer:

$$
\sigma_{m}=-\int_{0}^{\infty} d y \sum_{i} z_{i} e N_{i}(y)=-\left.\epsilon_{r} \epsilon_{0} \frac{d \psi(x)}{d x}\right|_{x=0} .
$$

\subsection{The Maximum Density Model}

The Boltzmann distribution, Eq. 1, predicts for a reasonable surface potential of $-50 \mathrm{mV}$ an interfacial $\mathrm{RuR}$ concentration of $1.2 \mathrm{M}$ which corresponds to $\approx 10^{27}$ molecules $/ \mathrm{m}^{3}$, assuming a bulk RuR concentration of $10^{-5} \mathrm{M}$. By comparing this number to the volume of RuR (using $a=1.2 \mathrm{~nm}$ and $r=0.2 \mathrm{~nm}$, one finds that all free space in front of the surface would have to be occupied by RuR. In order to get a rough estimate of how this extremely high ion density could affect the potential distribution, an empirical term was introduced which inhibits a further rise of the ion density at high potentials

$$
N_{M D}(x)=N(x) \exp \left(-\frac{N_{M D}(x)}{N_{\max }}\right),
$$

where $N_{\max }$ is the maximum density of ions estimated by their size and interaction radius which is assumed to be determined by the Debye length $\kappa^{-1}$

$$
N_{\max }=\left(\pi a \kappa^{-2}\right)^{-1}
$$

Substituting $1.2 \mathrm{~nm}$ for the length of RuR and a Debye length of $2.5 \mathrm{~nm}^{9}$ yields a maximum concentration (converted to moles/liter) of about $0.07 \mathrm{M}$ which is small

\footnotetext{
${ }^{8}$ see Appendix B.3

${ }^{9}$ determined mostly by the buffer concentration used in experiments (Völker and Smejtek, 1995)
} 
compared to that predicted by the pure Boltzmann distribution in front of the membrane surface.

As the adsorbed ions are not completely removed from the aqueous phase one should take their presence in Eq. 13 into account. It was found that the correction is negligible within our error limits.

Numerical procedures for solving the model are given in Appendix B.4.

\subsection{The Adsorption Model}

In this section a brief outline of the model used by Völker and Smejtek (1995) to fit their experimental data is presented. The adsorption of RuR to lipid vesicles has been measured according to

$$
R_{\mathrm{RuR}}=\frac{[\mathrm{RuR}]_{e q}}{[\mathrm{RuR}]_{\text {init }}}
$$

where $[\mathrm{RuR}]_{e q}$ is the equilibrium aqueous concentration of RuR in the presence of liposomes and $[\mathrm{RuR}]_{\text {init }}$ is the initial concentration before adding the liposomes to the aqueous phase. $R_{\mathrm{RuR}}$ varies between 0 and 1 ; the smaller $R_{\mathrm{RuR}}$, the greater the adsorption. The dependence of $R_{\mathrm{RuR}}$ on $[\mathrm{RuR}]_{\text {init }}$ is defined as the distribution isotherm. The equilibrium concentration $[\mathrm{RuR}]_{e q}$ depends on the adsorption of RuR to the bilayer, namely on the membrane surface density of RuR, $(\mathrm{RuR})_{m}$. For bilayer prepared from two types of phospholipids: electrically neutral PC (L- $\alpha$ phosphatidylcholine) and negatively charged $\mathrm{PX},{ }^{10}$ the balance of free and bound RuR ions, is given by

$$
[\mathrm{RuR}]_{e q}+(\mathrm{RuR})_{m} A_{\text {lip }}=[\mathrm{RuR}]_{\text {init }} .
$$

$A_{l i p}$, the surface area per unit volume of liposome suspension, is given by

$$
A_{l i p}=P_{P X}[P X]+P_{P C}[P C],
$$

\footnotetext{
${ }^{10} \mathrm{PX}$ represents one of the charged lipids: PS ( $\mathrm{L}-\alpha-$-phosphatidylserine), PI ( $\mathrm{L}-\alpha-$ phosphatidylinositol) or PG (L- $\alpha$-phosphatidylglycerol).
} 
where $P_{P X}$ and $P_{P C}$ designate the area per lipid of negatively charged and neutral lipids, and $[\mathrm{PX}]$ and $[\mathrm{PC}]$ are their respective concentrations in the suspension of liposomes. A similar balance equation is needed for the monovalent cations (CAT). In modeling RuR adsorption it was assumed that the concentration of monovalent cations in water did not appreciably change ${ }^{11}[\mathrm{CAT}]_{e q} \approx[\mathrm{CAT}]_{\text {init }}$.

Competitive adsorption of monovalent cations and $\mathrm{RuR}$ to the bilayer describable by a Langmuir adsorption isotherm is assumed. The surface density of RuR ions, $(\mathrm{RuR})_{m}$, depends therefore upon the association constant, $K_{\mathrm{mRuR}}$, the concentration of $\mathrm{RuR}$ adjacent to the membrane, $[\mathrm{RuR}]_{i f}$, and the density of free sites available to RuR cations, $f_{R u R}$. A similar equation holds for monovalent cations, such as potassium:

$$
(\mathrm{RuR})_{m}=K_{m R u R}[\mathrm{RuR}]_{i f} f_{R u R} \quad(\mathrm{CAT})_{m}=K_{m C A T}[\mathrm{CAT}]_{i f} f_{C A T} .
$$

As we assume competitive adsorption to negatively charged lipids only, we have a surface density of free adsorption sites $f_{R u R}=f_{C A T}=f$, which is related to the total density of sites, $Q_{P X}$, by

$$
(\mathrm{RuR})_{m}+(\mathrm{CAT})_{m}+f=Q_{P X}
$$

where $Q_{P X}$ is determined by the concentration of negatively charged lipids,

$$
Q_{P X}=\frac{[P X]}{A_{l i p}} .
$$

A Boltzmann relation is used to relate the interfacial concentrations of adsorbing species to their concentrations in the bulk solution

$$
\frac{[\mathrm{RuR}]_{i f}}{[\mathrm{RuR}]_{e q}}=\exp \left(\frac{-z_{R u R} e \psi(0)}{k T}\right) \quad \frac{[\mathrm{CAT}]_{i f}}{[\mathrm{CAT}]_{e q}}=\exp \left(\frac{-e \psi(0)}{k T}\right) .
$$

Völker and Smejtek (1995) had to introduce an effective charge for RuR $z_{R u R \text {,eff }}<$ $z_{R u R}$ to account for effects not included in the GC Model. It is the purpose of this study to find a model in which RuR keeps its physical valency of six.

\footnotetext{
${ }^{11}$ This simplification is justifiable in view of the rather small binding constant of potassium ions to negatively charged lipids $\left(\approx 1 \mathrm{M}^{-1}\right)$ and the experimental conditions.
} 
The membrane surface charge density, $\sigma_{m}$, depends on the charge of the native PC-PX membrane, $\sigma_{0}$, and on the surface densities of adsorbed cations,

$$
\sigma_{m}=\sigma_{0}+e(\mathrm{CAT})_{m}+6 e(\mathrm{RuR})_{m}
$$

The theoretical distribution isotherm $R_{\mathrm{RuR}}$ is obtained by solving the set of equations, Eqs. 15-22, with Eq. 4 and Eq. 3 for GC, Eq. 11 together with Eq. 12 for the Rod Model and Eq. 13 together with Eq. 14 for the Maximum Density Model. Due to its long calculation time, the Rod Model was solved using a grid of only 500 intervals for the integration. Numerical procedures are described in Appendix B.5. 


\section{Chapter 3}

\section{Results and Discussion}

\subsection{Electrostatic Calculations}

The electrostatic calculations were performed assuming a $p H$ of 7.3 in $0.001 \mathrm{M}$ citrate/borate/phosphate $(2 \mathrm{mM} / 2 \mathrm{mM} / 0.5 \mathrm{mM})$ buffer $\left(\mathrm{B}^{-3}\right)$. The dielectric constant of the aqueous solution was assumed to be $\epsilon_{r}=78.5$.

In order to get a picture of the specific model properties, both the potential distribution (Figure 8) and the Boltzmann factors (Figure 9) have been evaluated. A higher bulk RuR concentration of $0.01 \mathrm{M}$ has been chosen to exaggerate the characteristics of the models which become more evident at higher concentrations.

Exponential decay of the potential is common to all models. Characteristic for the Rod Model is the steep slope in the interfacial region and a less decaying asymptotical behavior which leads to a crossing-over with the other potentials (see arrow in Figure 8). Two opposing processes might explain this behavior: (i) the surface potential decreases almost exponentially resulting in a decrease of the Boltzmann factor, and (ii) the number of available orientations for the rod increases with distance from the surface resulting in an increase of the Boltzmann factor. This effect can clearly be seen for charge B which has no possible orientations at the surface resulting in a vanishing Boltzmann factor for $x=0$. In the early stages of the iteration 


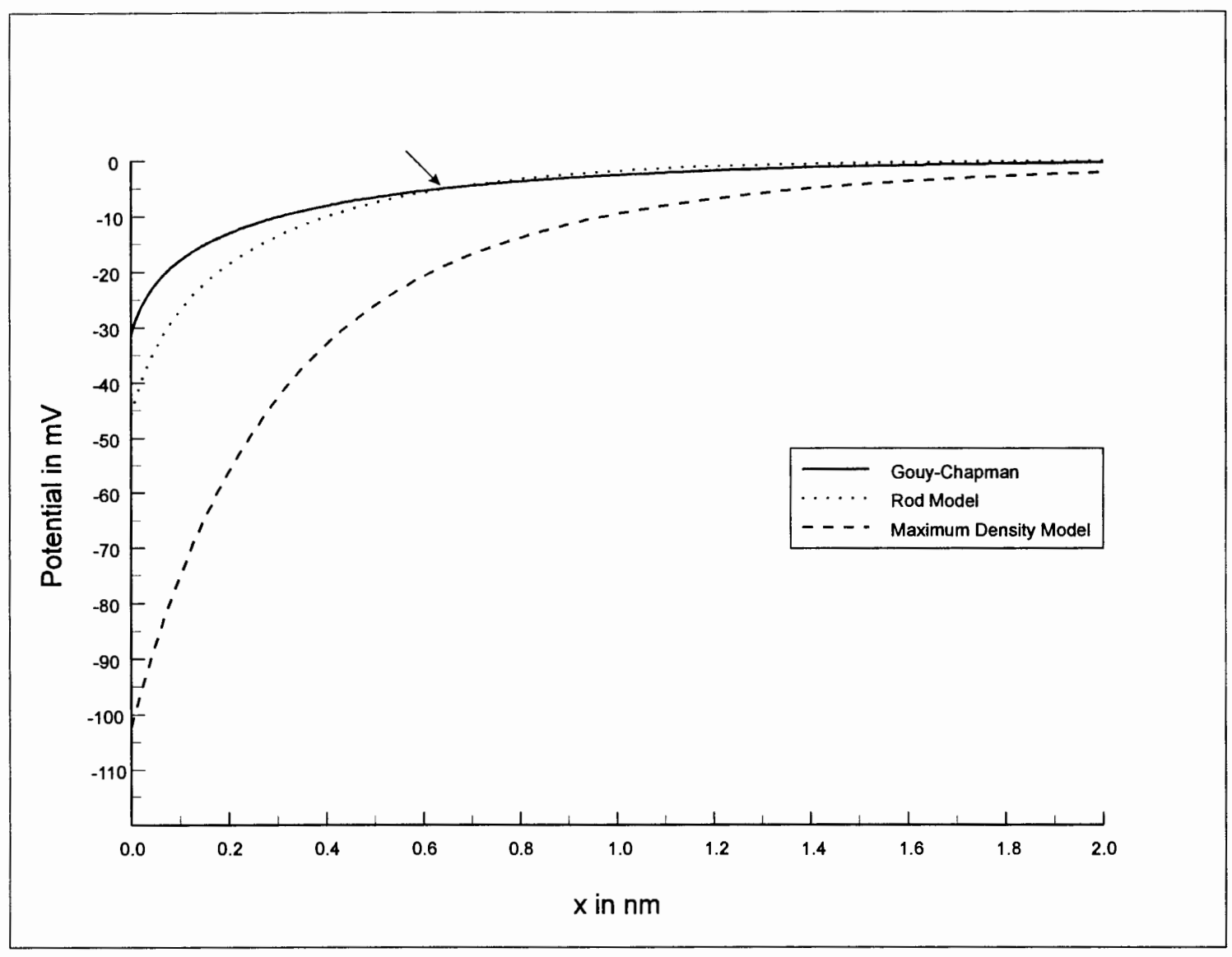

Figure 8: Potential distributions for the three studied models. Surface charge density $1.4 \mathrm{e} / \mathrm{nm}^{2}$, RuR bulk concentration $0.01 \mathrm{M}, p H 7.3$, buffer $\mathrm{B}^{-3}$, Debye length 0.64 $\mathrm{nm}$; the arrow marks the crossing-over of the potential calculated by the Rod Model and of that calculated from the GC Model. 


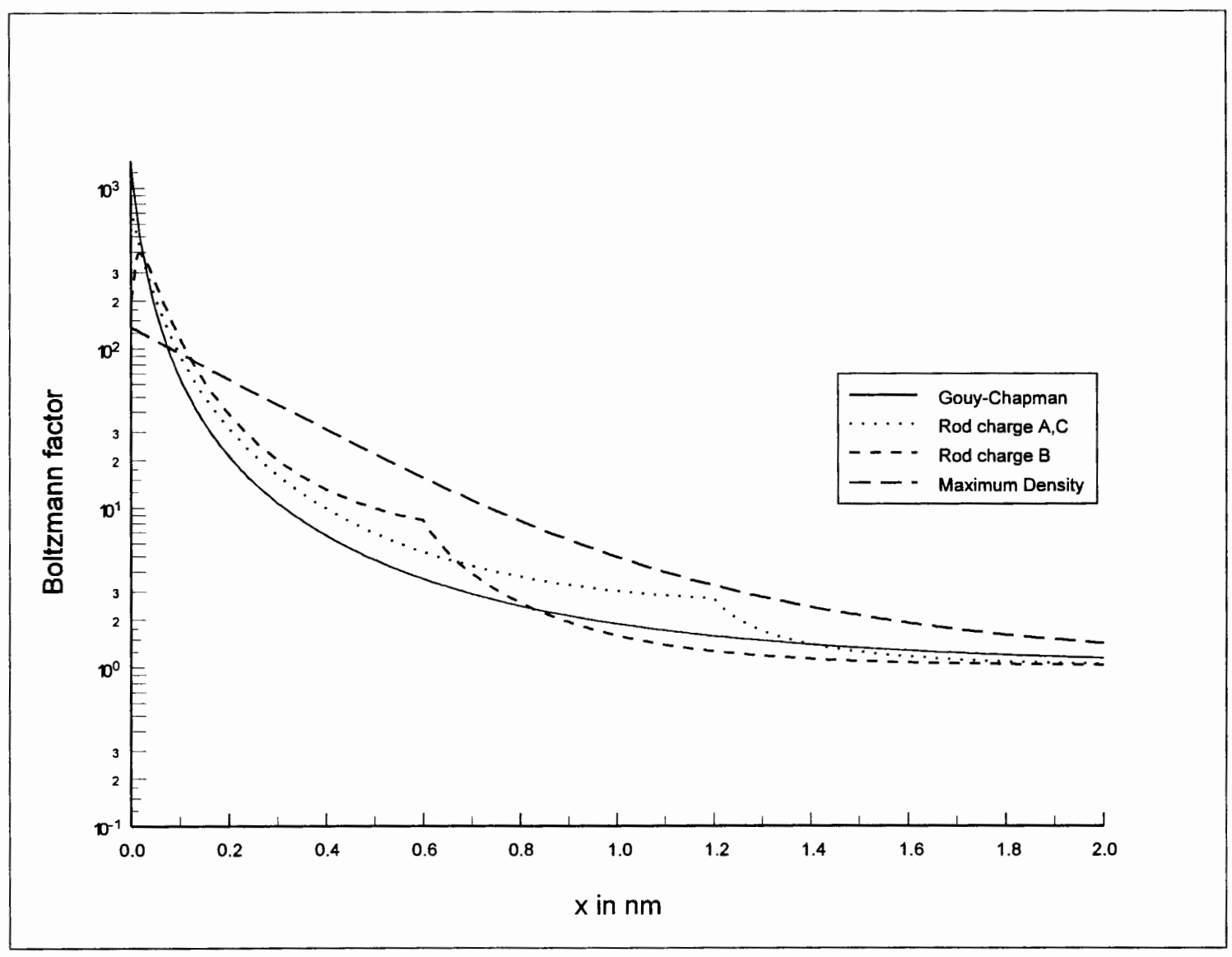

Figure 9: Boltzmann factors for the three studied models. Parameters are the same as in Figure 8. 
of the surface potential, the increase of available states supercedes the decrease of the potential and the Boltzmann factor actually increases for $x \lesssim a_{0}$, where $a_{0}$ is equal to the length or half the length of the rod for charges type A,C or B, respectively. This might be considered as an energetically unfavorable state and therefore the slope of the potential increases to maintain the Boltzmann factor decaying monotonically. An increase in slope, on the other hand, decreases the integral number of ions in the double layer which leads to a violation of the electroneutrality condition, Eq. 3. To compensate for this, the surface potential rises in magnitude due to less efficient screening. The abrupt change of the slope for the Boltzmann factors at position $a_{0}$ arises from the fact that from this position on, the rod can occupy all available orientations no longer constrained by the membrane.

The potential distribution for the Maximum Density Model exhibits a very large surface potential but similar slope compared to GC. This can also be explained in terms of the electroneutrality condition. As the model effectively decreases RuR concentration (by at least an order of magnitude) in the wider interfacial region, the surface potential increases to maintain charge neutrality in the diffuse double layer which in turn increases the Boltzmann factor resulting in a reduced effect on $[\mathrm{RuR}]_{i f}$.

Lower bulk concentrations of RuR result in smaller deviations of the Boltzmann factors and potentials from GC (not shown).

To relate the Boltzmann factors to an effective charge for RuR, the effective valency in GC was varied until the Boltzmann factor matched the one for the Rod or the Maximum Density Model. Figure 10 shows the results for the Rod Model and Figure 11 for the Maximum Density Model for different RuR concentrations. The figures include experimentally obtained effective valencies from adsorption studies (Völker and Smejtek, 1995).

The effective valency plots have to be interpreted very carefully as they cannot be compared directly to the experimental values which were obtained from fits of the Adsorption Model employing different bulk concentrations of RuR. 


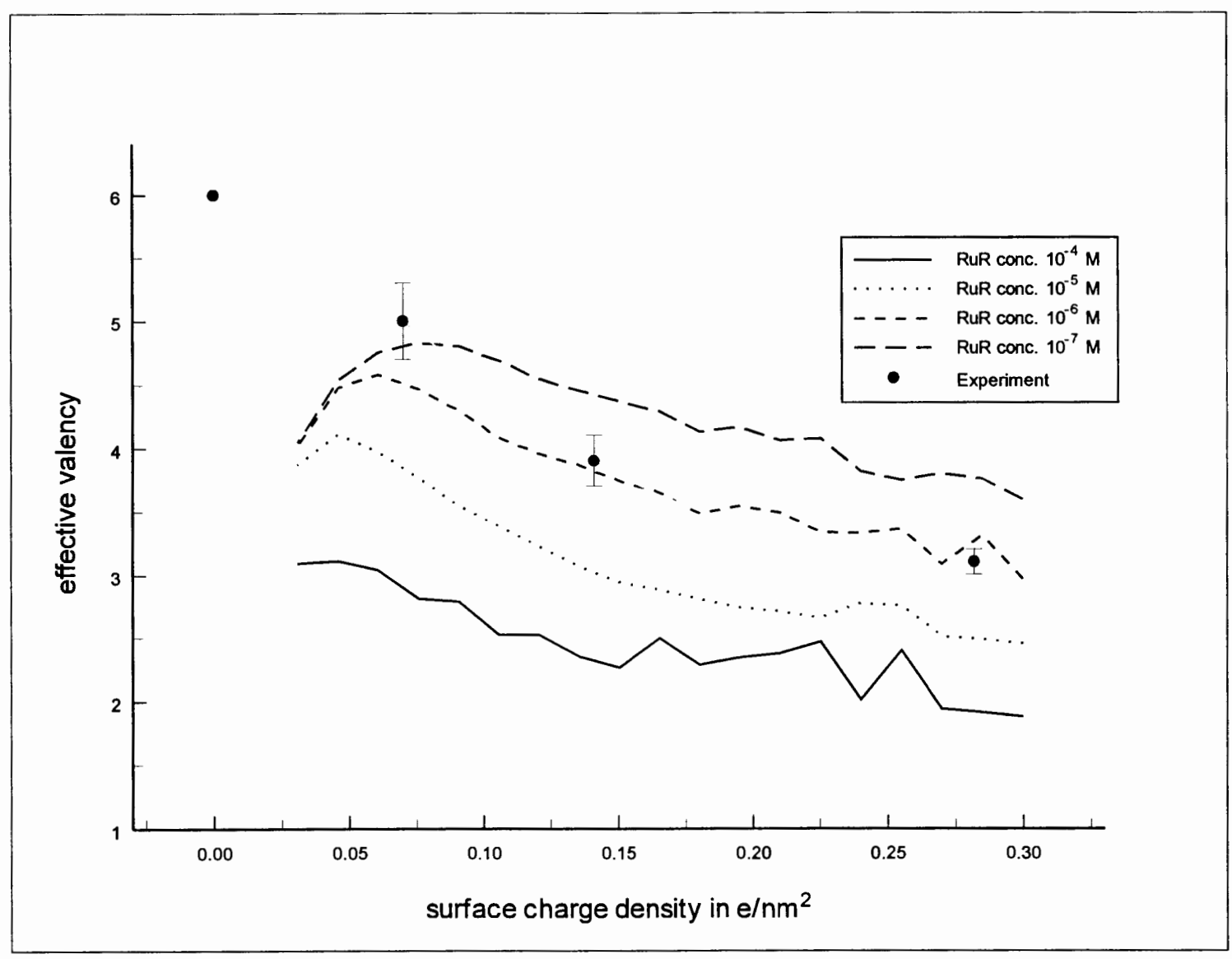

Figure 10: Effective valency for Gouy-Chapman to fit the Rod Model. 


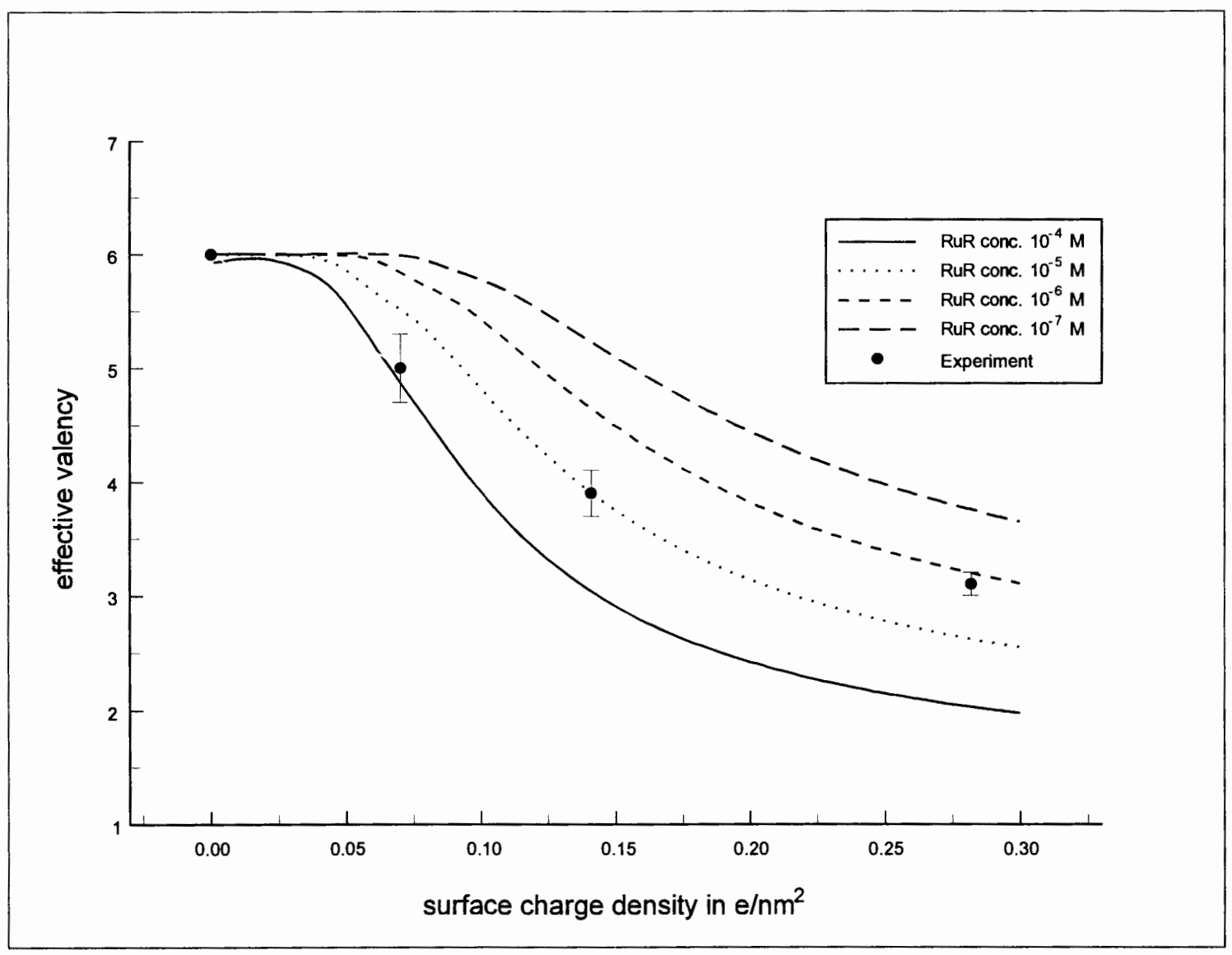

Figure 11: Effective valency for Gouy-Chapman to fit the Maximum Density Model. 
The figures show that both models reflect the tendency of the experimental data: decreasing surface charge density results in increasing valency with an asymptotic approach of the physical value of six.

It is important to note that the Rod Model fails for small surface charge densities. The reasons for this and the deviation from a smooth curve are still unknown. A failure of the algorithm can be excluded as calculations with increased accurracy gave the same result.

\subsection{Adsorption Isotherm Calculations}

The Rod and the Maximum Density Model can be applied to the Adsorption Model. The calculations for the Adsorption Model were performed with an association constant for potassium ions $K_{m C A T}=1$, an association constant for Ruthenium Red ions $K_{m R u R}=5.5$ and an area per lipid of $0.71 \mathrm{~nm}^{2}$. Figure 12 shows the adsorption isotherms for a lipid composition of $\mathrm{PC}: \mathrm{PS}=9: 1$. The plots represent calculated values for the GC Model assuming a valency of 6 and 3.9 and for the Rod Model with valency 6. A plot for the Maximum Density Model has been omitted as it would be indistinguishable from the GC valency 6 plot which originates from the similarity of their interfacial RuR concentrations (Figure 14). This similarity arises from the fact that $[\mathrm{RuR}]_{\text {if }}$ is so low $\left(10^{-6}-10^{-4} \mathrm{M}\right)$ that ion interactions do not play an important role. $^{1}$

The Rod Model improves the fit to the data substantially when compared to GC with valency 6 . This improvement is even better for lower surface charge densities (PC:PS=9:1) as can be seen from Figure 13 in which the effective valency for the GC fit was set to 3.1 .

Nevertheless the model overestimates the RuR adsorption for lower initial bulk

\footnotetext{
${ }^{1}$ the maximum density calculated according to Eq. 14 for our experimental conditions is about $0.07 \mathrm{M}$
} 


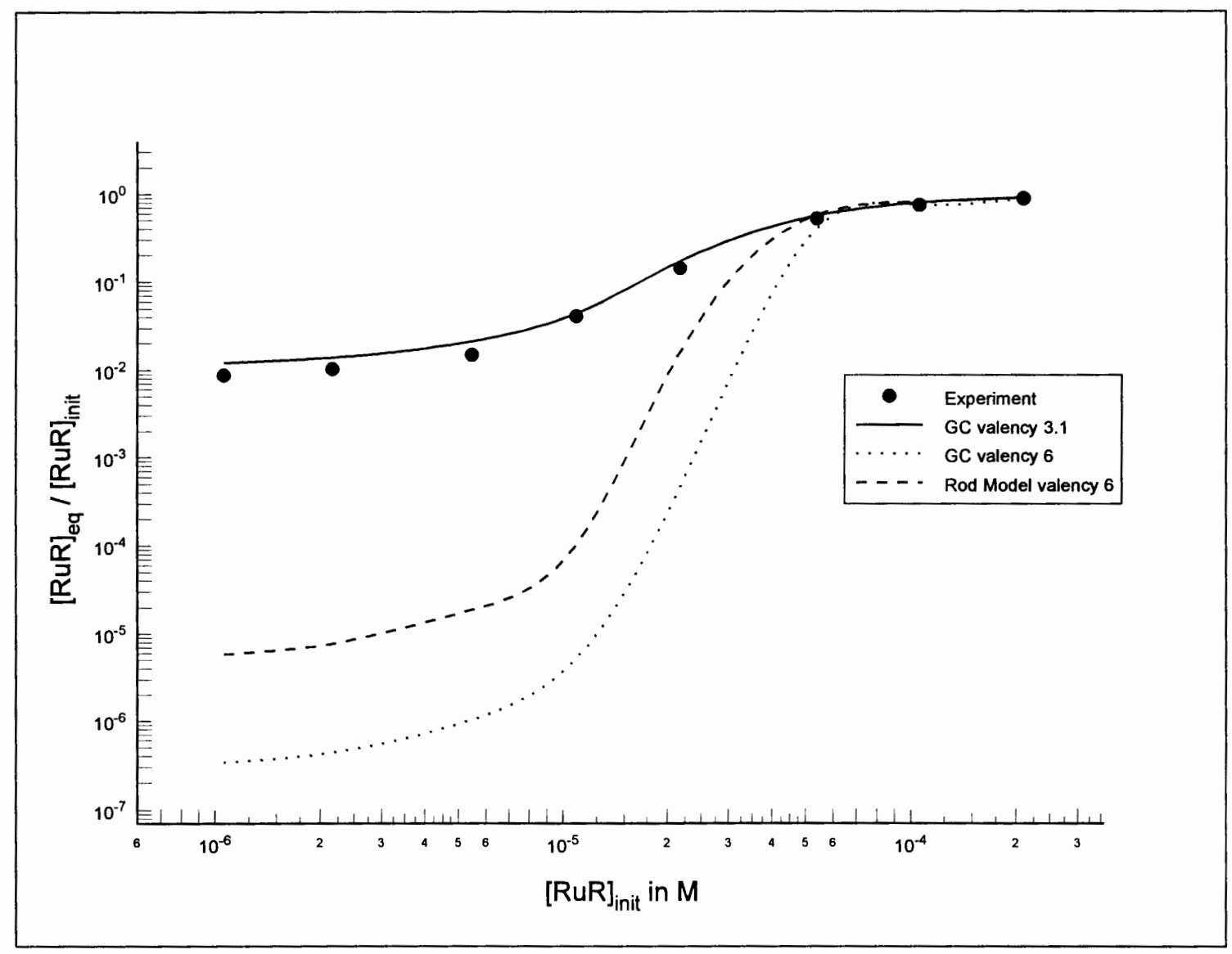

Figure 12: Langmuir adsorption isotherm for RuR adsorption to PC:PS=4:1 lipid vesicles, $p H 7.3$, buffer $\mathrm{B}^{-3}$. 


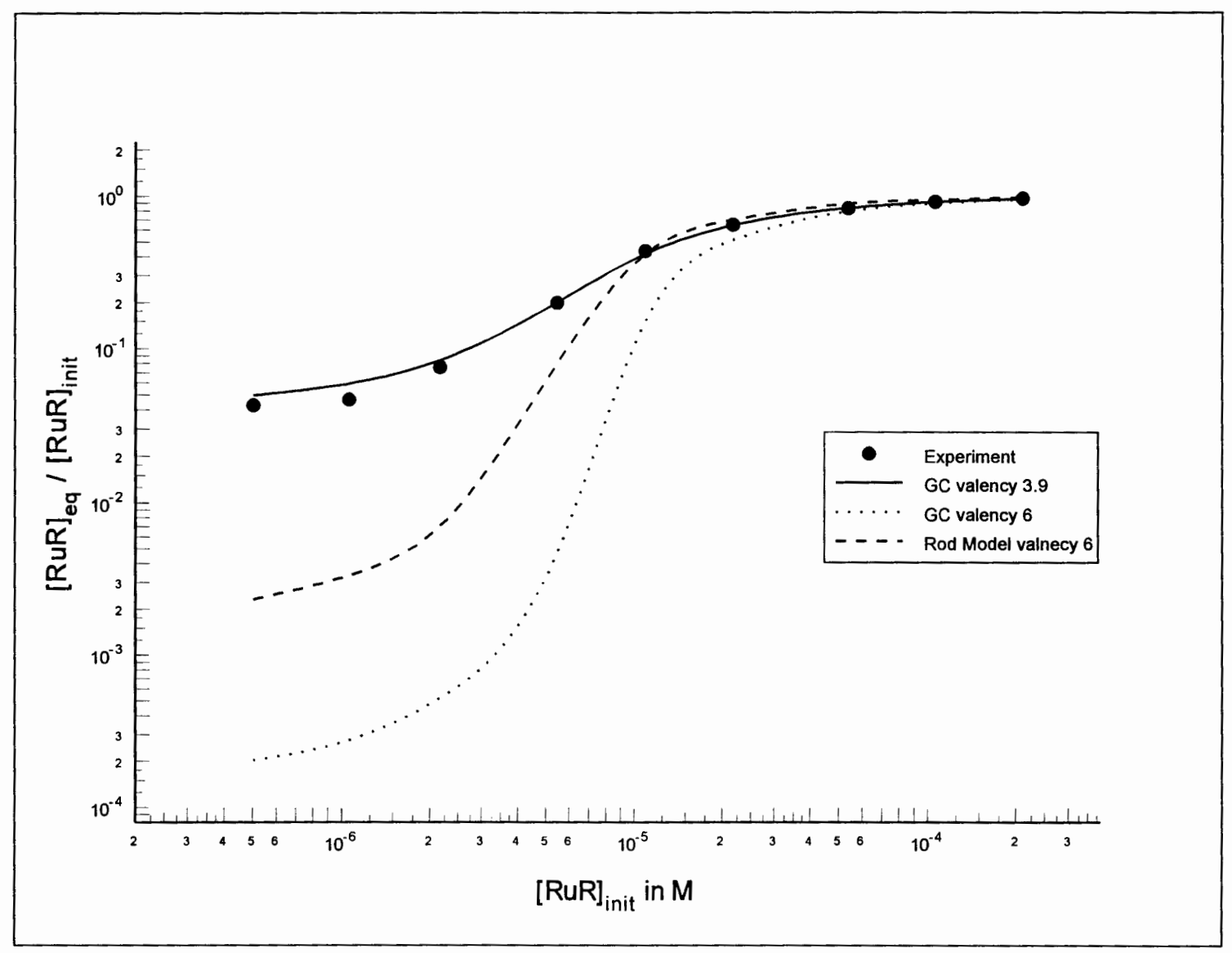

Figure 13: Langmuir adsorption isotherm for RuR adsorption to $\mathrm{PC}: \mathrm{PS}=9: 1$ lipid vesicles, $p H 7.3$, buffer $\mathrm{B}^{-3}$. 


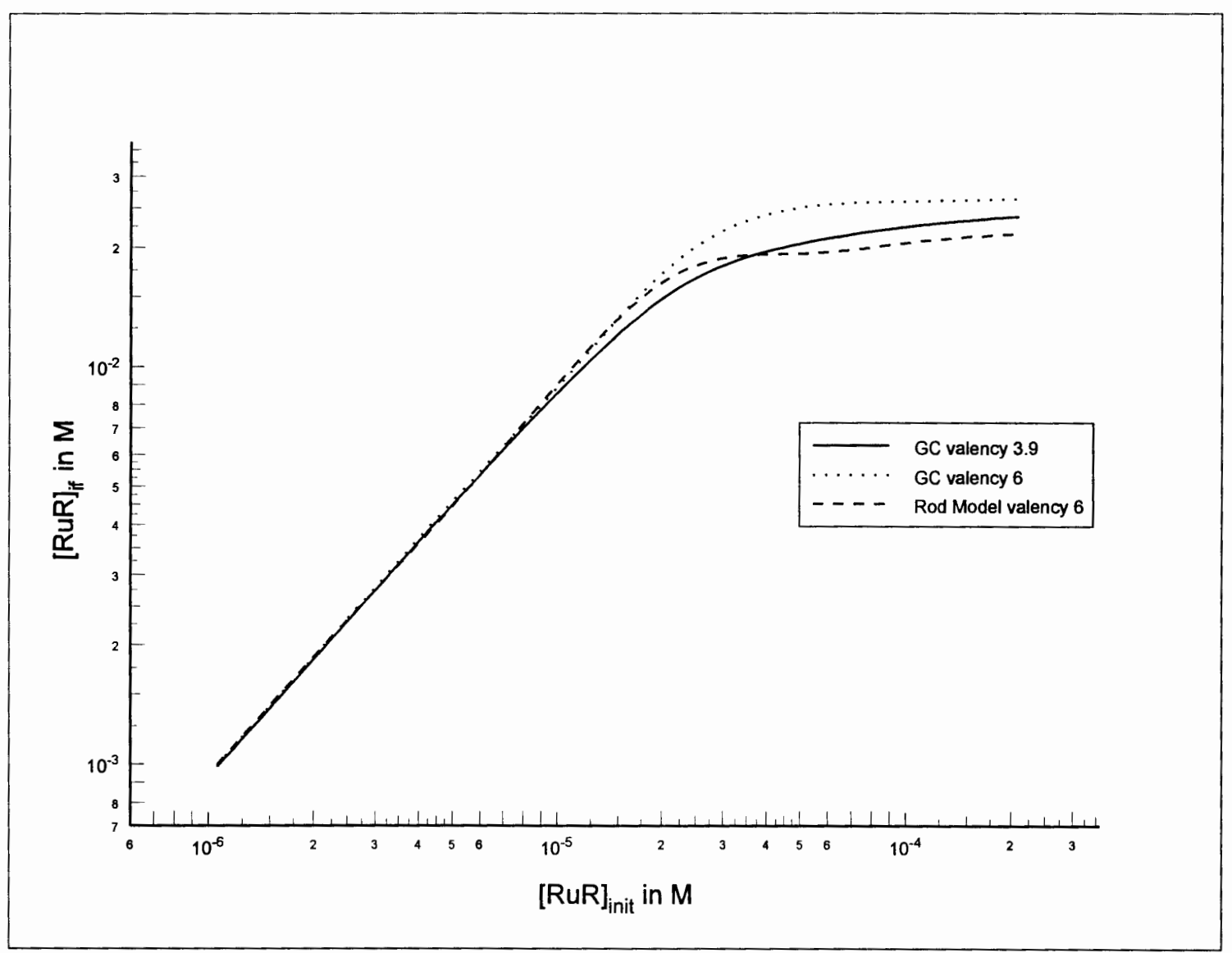

Figure 14: Interfacial RuR concentration for PC:PS=4:1, $p H$ 7.3, buffer $\mathrm{B}^{-3}$. 
concentrations. It is remarkable though that the difference in interfacial RuR concentration (Figure 14) is miniscule for low initial RuR concentrations and increases for higher concentrations where the model seems to predict the correct adsorption. The reason for this lies in the saturation of the adsorption isotherm ${ }^{2}$ at high concentrations reducing the influence of the interfacial RuR concentration on the overall adsorption. For lower concentrations on the other hand, a minute increase in $[R u R]_{i f}$ results in an increase in adsorption which leads to a depletion of the bulk phase as the surface area available for adsorption ${ }^{3}$ is around $500 \mathrm{~m}^{2}$. One might argue that e.g., for the Maximum Density Model, the concentration of adsorbed ions needs to be included in the interfacial concentration as it is likely that adsorbed ions protrude into the aqueous phase from the membrane surface and therefore contribute to the maximum density. But due to the huge surface area, the concentration of adsorbed ions is negligible compared to $[\mathrm{RuR}]_{i f}$ (data not shown).

\subsection{Conclusions}

In this theoretical study it has been shown that steric effects play an important role in the spatial distribution of Ruthenium Red ions. Obtaining a new set of adsorption parameters using these models would be desirable but is not feasible due to the long computation times.

The results of the Maximum Density Model show that despite the failure of the model to correctly predict the adsorption isotherm, ion-ion interactions which are not included in the GC Model, are expected to be important at charged membrane surfaces.

To explore a possible way to incorporate ion-ion interactions one might consider a model in which the discreteness of the surface charge is included. Discreteness of charge gives rise to high ion concentrations in front of the charged lipids. A

\footnotetext{
${ }^{2}$ the high concentration of RuR adsorbed to the membrane decreases the surface charge density resulting in a substantial decrease of the surface potential

${ }^{3}$ area calculated for $1 \mathrm{~g}$ lipid solution $\left(\mathrm{MW} \approx 800 \mathrm{u}\right.$ ) assuming a lipid area of $0.71 \mathrm{~nm}^{2}$
} 


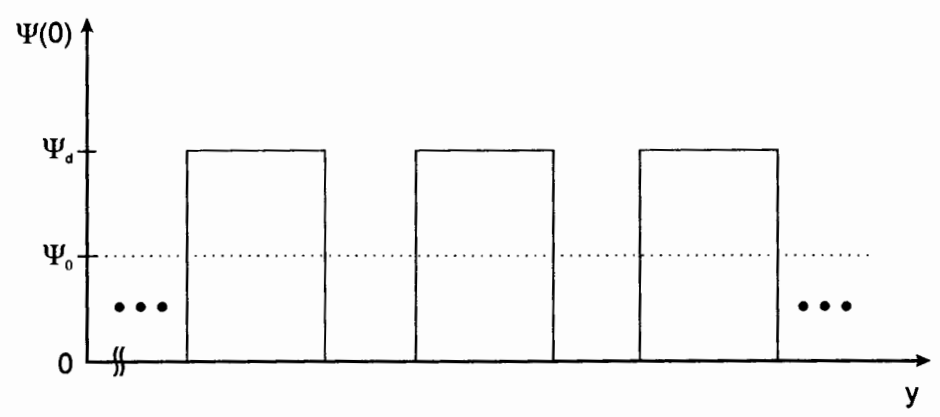

Figure 15: Primitive Discrete Charge Model. $\psi_{d}$ discrete potential of the reduced area, $\psi_{0}$ average potential.

primitive approach to model the discreteness of the charges is to use a rectangular potential, as shown in Figure 15. Neglecting adsorption and electrostatic interactions at the areas of vanishing potential, this model simply reduces the active surface area associated with an increase in surface potential obtained by assuming a constant average potential

$$
\psi_{d}=\frac{A_{0}}{A_{d}} \psi_{0}
$$

where $\psi_{d}$ denotes the new surface potential, $\psi_{0}$ the initial average potential, $A_{0}$ the initial surface area and $A_{d}$ the area remaining after subtraction of the inactive area. Assuming a ratio of $A_{0}: A_{d}=2: 1^{4}$, leads to a doubling of the potential and a squaring of the Boltzmann factor resulting in an interfacial RuR concentration of about $75 \mathrm{M}$ compared to $1 \mathrm{mM}$. At this concentration ion-ion interactions certainly become important. Applying the Maximum Density Model, one obtains a lower boundary for the concentration $N_{M D}=0.07 \mathrm{M}$ (the maximum density Eq. 14). As the surface area in reality is twice as large, the concentration halves to $0.035 \mathrm{M}$. A rough estimate might suggest that the adsorption due to the decrease of binding sites also decreases by a factor of two. But even with this assumption the adsorption will be much higher then before. In addition to that, increasing charge density should

\footnotetext{
${ }^{4}$ which is a reasonable assumption considering the interlipid distance on the basis of a hexagonal lattice
} 
decrease the effect. We observe a smaller deviation for our models with an increase in surface charge density. It is therefore unlikely that discrete charge effects play an important role.

From the properties of the two models and the obtained results, conclusions for the possible characteristics of a more complete model in which RuR has its physical valency of six can be drawn. Within the framework of the smeared charge theory, steric effects are fully accounted for in the Rod Model. The failure of the Maximum Density Model is twofold: (i) the fulfillment of the charge neutrality condition leads to a substantial decrease of its effectiveness to reduce interfacial concentration, and (ii) low interfacial concentrations in the Adsorption Model suggest the absence of ion-ion interactions.

To overcome these failures one would need a much stronger nonlinear process which would alter only the ion distribution in front of the surface keeping the surface potential close to the GC result. This demands a steeper slope of the spatial distribution of the potential in the interfacial region as observed for the Rod Model.

The only way to account for these nonlinearities would be the application of one of the statistical mechanical approaches which incorporate "real" ion-ion interactions. Stankowski's (1991) Virial Expansion Model is a promising candidate for future explorations. Integral Equation Models like HNC would certainly be the most suitable tool for the problem, but due to their difficult implementation their use in experimental studies is limited. 


\section{Part II}

\section{Distribution of}

$2,3,4,5$-Tetrachlorophenol in a Membrane Model System 


\section{Chapter 4}

\section{Introduction}

Chlorinated phenols have been extensively employed in different industrial and agricultural applications. Pentachlorophenol (PCP) was the most widely used chlorinated phenol, but other chlorinated phenols like tetrachlorophenol (TeCP) and trichlorophenol (TrCP) are side products of chemical reactions in chlorobleaching of Kraft pulp (Paasivirta et al., 1992) and evolve during the breakdown process of PCP by UV light and biodegradation (Murthy et al., 1979; Mikesell and Boyd, 1986). Therefore they can be found in the environment in concentrations similar to that of PCP (Butte, 1985; Rogers et al., 1992). Wood treatment was the major application of the 23 million kilograms of pentachlorophenol produced yearly in the U.S. (Cirelli, 1978; Dougherty, 1978) that ultimately end in the environment. Due to their strong oxidative character chlorinated phenols have also been extensively

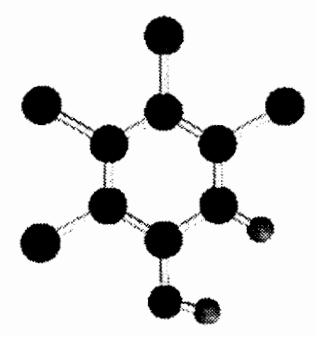<smiles>OC1CC(Cl)C(Cl)C(Cl)C1Cl</smiles>

Figure 16: Molecular structure of 2,3,4,5-tetrachlorophenol (TeCP). 
used as biocides to control bacteria, fungi, mollusks, insects, slime, and other biota (Kaufman, 1978). They have long been known to disrupt cell function by destroying the proton gradient across the inner mitochondrial membrane and therefore uncoupling the oxidative phosphorylation of ATP (Weinbach, 1956). Partitioning of the hydrophobic chlorinated phenols into the lipid phase of the bilayer (Buff et al., 1982) enables proton transport across the membrane (Jayaweera et al., 1982). ${ }^{1}$ It has also been shown that their presence decreases the fluidity of lipid bilayers (Duxbury and Thompson, 1987), the gel-to-fluid phase transition temperature (Smejtek et al., 1989), the free amino acid pool in freshwater amphipod Gammarus pseudolimnaeus (Graney and Giesy, Jr., 1987) and the carbon assimilation by algae (Jayaweera et al., 1982). For an overview of the membrane toxicity of PCP see Smejtek (1987).

Although the production of PCP has been discontinued or its use reduced in many countries, it is believed that chlorinated phenols continue to be one of the major pollutants in the environment since they are already ubiquitous.

Several studies and estimates in Germany (Geyer et al., 1987), the United States (Hattemer-Frey and Travis, 1989) and the United Kingdom (Wild and Jones, 1992) showed that the food chain is the major source (90-99\%) of PCP exposure to humans along with inhalation of vapors from treated wood and the consumption of drinking water, which accumulate to an estimated long-term average daily intake of about 10$48 \mu \mathrm{g} /$ day. The average body burden of PCP varies for different countries between $548 \mu g$ and $673 \mu g$.

Although it has been shown (Geyer et al., 1987) that the half-life of PCP in humans is about 19 days, there is strong evidence for chronic effects due to long term exposure (Crosby, 1981) especially in organs with high bioconcentration factors $(\mathrm{BCF})$ which are liver $(\mathrm{BCF}=5.7)$ and brain $(\mathrm{BCF}=3.3)$ (Geyer et al., 1987). The

\footnotetext{
${ }^{1}$ there are two possible mechanisms: (i) $\mathrm{A}^{-}$penetrates through the membrane, binds to a $\mathrm{H}^{+}$, diffuses back and releases the proton to the outside (Terada, 1990) or (ii) an AHA- complex is formed at the outer surface, permeates through the membrane, binds a proton which results in a dissociation of the dimer, $\mathrm{AH}$ travels back to the outer surface of the membrane and releases the proton (Barstad et al., 1993)
} 
high hydrophobicity of chlorinated phenols leads to a strong affinity to lipid material such as cell membranes or organic material in soils to which they adsorb.

Kerler and Schönherr (1988), among others, showed that the ability of substances to adsorb to organic material is closely related to their octanol/water partition coefficient, $P_{O W}$.

$P_{O W}$ is most commonly obtained by the shake flask method as proposed by OECD (OECD, 1981). In this approach a compound $\mathrm{X}$ is injected into a two-phase system consisting of known volumes of octanol and water. After shaking the solution and separating the phases, samples from either the water phase and/or the octanol phase are taken and the concentration of the compound is determined. The octanol/water partition coefficient $P_{O W}$ is defined by

$$
P_{\text {ow }}=\frac{[\mathrm{X}]_{o}}{[\mathrm{X}]_{W}},
$$

where the subscript "O" stands for the octanol and " $\mathrm{W}$ " for the aqueous phase. As the procedure is very laborious, ${ }^{2}$ other methods of obtaining $P_{O W}$ have been explored. One of them is the generator column method invented by Wasik et al. (1981) and improved for $\log P_{O W}$ values $>6$ by Woodburn (1984). In the slow stirring method the solution is not shaken but slowly stirred under close temperature control to avoid emulsion formation (Brooke et al., 1986). It is especially designed for very high values of $P_{O W}$ but requires long stirring times (2-3 days) to reach equilibrium.

There are several indirect methods, experimental as well as numerical, to estimate $P_{o W}$. The most frequently used is the estimation of $P_{o W}$ based on reversedphase high-performance liquid chromatography (RP-HPLC) retention times in which a linear relationship between retention times of known compounds and their partition coefficient is used to predict $P_{O W}$ of a unknown compound (Weber, Jr. et al., 1986). Probably the least accurate estimate for the partition coefficient can be obtained from the water solubility of a compound (Miller and Wasik, 1985) using the

\footnotetext{
${ }^{2}$ each sample point requires about 4 hours
} 
relation

$$
\begin{aligned}
& \log P_{\text {OW }}=A-B V-\log C_{L}^{S} \quad \text { for liquids and } \\
& \log P_{\text {OW }}=A-B V-\log C_{S}^{S}+\log F \quad \text { for solids, }
\end{aligned}
$$

where $V$ is the molar volume, $F$ the fugacity ratio given by $F \approx \exp \left[6.8\left(1-T_{M} / T\right)\right]$ with the melting temperature $T_{M}, C_{L}^{S}$ and $C_{S}^{S}$ are the water solubilities for liquid and solid compounds, respectively, and $A$ and $B$ are correlation parameters obtained from experimental data.

Several numerical estimation methods for the partitioning of compounds into organic matter using the octanol/water partition coefficient have been established. Quantitative structure activity relationships (QSAR) are the most accurate approach in which characteristics of the compound like charge density distribution, number of different atoms and functional groups are accounted for in simple regression equations of the form

$$
\log P_{\text {ow }}=\text { const. }+\sum_{i} n_{i} P_{i}+\sum_{i} P_{i}^{\prime} q_{i}+\sum_{i} P_{i}^{\prime \prime} q_{i}^{2}+\sum_{m} F_{m}
$$

where $P_{i}, P_{i}^{\prime}, P_{i}^{\prime \prime}$ are parameters characteristic of a given atom $i, n_{i}$ is the number of occurences of such atoms and $q_{i}$ is the charge density on $i . F_{m}$ represents the presence or absence of functional groups (Leo et al., 1971; Klopman et al., 1985). Extensions including solvatochromic parameters, ${ }^{3}$ molecular surface, and volume (Kamlet et al., 1988; De Bruijn and Hermens, 1990) improved the correlation of data. Similar models have been proposed to calculate the partition coefficient for a new solute on the basis of a known coefficient. This opens the possibility for obtaining a set of parameters which relates structural and/or functional properties of molecules to their toxicity (Leo et al., 1971), bioconcentration (Mackay, 1982; van Gestel et al., 1985) and presence in soils and sediments (Schellenberg et al., 1984; Lagas, 1988). For a recent review of different QSAR methods see Sabljić (1991).

\footnotetext{
${ }^{3}$ solute polarizability, hydrogen bond acceptor basicity, and hydrogen bond donor acidity
} 
Although the octanol/water model is successful in predictions of partitioning of neutral molecules in biological tissue, the situation is different if ions are present in the system. This holds in particular for hydrophobic ionizable organic compounds (HIOC's) like chlorinated phenols (HA) with a $p K_{a}$ of between 3.7 and 8.3 for which ionized forms $\left(\mathrm{A}^{-}\right)$need most definitely to be taken into account at ambient $p H$ values. In this case two partition coefficients determine the distribution of the compound, one for the neutral form, $\gamma_{\mathrm{HA}}$, and one for the ionized form, $\gamma_{\mathrm{A}}$.

Kaiser (1982) studied the dependence of the apparent $P_{O W}$ of PCP as a function of $p H$ and determined significant anomalies which were believed to be partly caused by the varying ionic strength of the solution. Westall (1985) performed a detailed study of the effect of ionic strength and $p H$ on the distribution of chlorinated phenols in octanol/water. Smejtek and Wang (1993) compared partitioning of PCP in the octanol/water system with adsorption to phospholipid vesicles and found substantial differences for $p H>p K_{a}$.

The present study is in line with Smejtek's and Wang's studies. Our goal is the determination of the partition coefficient for 2,3,4,5-tetrachlorophenol and its phenolate, simultaneously, by means of the shake flask method. Different approaches for the theoretical description of the partitioning process are outlined and discussed. The obtained data are compared to results from adsorption studies for the same compound under the same experimental conditions (Blochel, 1992). The applicability of the octanol/water partition coefficient to predict biological significance in this particular case is questioned. 


\section{Chapter 5}

\section{Theory}

\subsection{Partitioning Theory}

The partitioning of a substance between two or more phases is described by the laws of thermodynamics. To derive a description for the state of a solute particle, one assumes an ideal gas (Adam et al., 1988) whose thermodynamic state is determined by its chemical potential, $\mu$, defined by ${ }^{1}$

$$
\mu=\left(\frac{\partial G}{\partial N}\right)_{T, V}
$$

where $N$ is the number of particles, $T$ the temperature, $V$ the volume and $G$ the free energy of the system, defined by

$$
G(N, V, T)=U+p V-T S=H-T S
$$

In this equation $S$ denotes the entropy, $U$ the internal energy and $p$ the pressure of the system. The latter quantities have been combined to the enthalpy $H=U+p V$. For a pure substance at constant temperature and volume, Eq. 28 reduces to

$$
\mu=\frac{G}{N}
$$

\footnotetext{
${ }^{1}$ the substripts denote the quantities held constant
} 
An infinitesimal change $d G$ at constant temperature results in a change $d \mu$ of the chemical potential which can be written as

$$
d \mu=\frac{d G}{N}=\frac{V}{N} d p=R T \frac{d p}{p}
$$

where $R$ is the ideal gas constant and the relation $p V=n R T$ for the ideal gas has been used. Integration of Eq. 31 assuming an initial pressure $p_{0}$ and initial chemical potential $^{2} \mu_{0}^{*}$ yields

$$
\mu=\mu_{0}^{*}+R T \ln \frac{p}{p_{0}}=\mu_{0}^{*}+R T \ln p
$$

where the initial pressure $p_{0}$ has been assumed to be 1 bar in the last step. $\mu_{0}^{*}$ is a constant determined by the initial pressure $p_{0}$, the temperature and the nature of the gas.

This result can easily be transferred to dilute solutions in which the interaction between the solute particles can be neglected as it was done for the ideal gas. Substituting the pressure $p$ in Eq. 32 by

$$
p=\frac{N}{V} R T=c R T,
$$

where $c$ is the molar concentration, gives the chemical potential of a single solute

$$
\mu=\mu_{0}^{*}+R T \ln (R T)+R T \ln c=\mu_{0}+R T \ln c .
$$

It is known from thermodynamic theory that chemical equilibrium is reached when $d G=0$. For the case of partitioning of a substance between two phases, $d G$ is given by the sum of the individual free energies of the solute in the two phases, $d G^{\prime}$ and $d G^{\prime \prime}$, which equals

$$
d G=d G^{\prime}+d G^{\prime \prime}=\mu^{\prime} d N^{\prime}+\mu^{\prime \prime} d N^{\prime \prime}=\left(\mu^{\prime}-\mu^{\prime \prime}\right) d N^{\prime},
$$

where a closed system ${ }^{3}$ has been assumed. The equilibrium condition $d G=0$ can only be fullfilled for $\mu^{\prime}=\mu^{\prime \prime}$ leading to

$$
\frac{\left(\mu_{0}^{\prime}-\mu_{0}^{\prime \prime}\right)}{R T}=\ln c^{\prime \prime}-\ln c^{\prime}=\ln \frac{c^{\prime \prime}}{c^{\prime}} .
$$

\footnotetext{
${ }^{2}$ often called the "standard chemical potential"

${ }^{3}$ conservation of particles: $d N^{\prime}=-d N^{\prime \prime}$
} 
This equation can be rewritten as

$$
P=\frac{c^{\prime \prime}}{c^{\prime}}=e^{\left(\mu_{0}^{\prime}-\mu_{0}^{\prime \prime}\right) / R T}=e^{-\Delta G_{0} / R T},
$$

where the partition coefficient $P$ and the free energy of transfer, $\Delta G_{0}$, have been introduced.

The theoretical determination of the partition coefficient has now been reduced to find an expression for the standard energy of transfer which is the energy needed to bring one mole of a substance from one phase to the other. According to Eq. 29 the free energy of transfer can be divided into an enthalpy term, $\Delta H$, and an entropy term, $T \Delta S$.

This general theory holds for partitioning of a substance in octanol and sorption to membranes. Differences arise from the fact that a membrane is a thin $(\approx 70 \AA)$ bilayer with a large surface area ${ }^{4}$ in contact with the surrounding solution, whereas octanol forms a bulk phase.

Partitioning into membranes is often described by the total energy model invented by Flewelling and Hubbell (1986). In this model the total energy of transfer ${ }^{5}$ is composed of the Born, image, dipole and neutral energy terms. The Born energy is the sum of energies needed to discharge a sphere in one dielectric, transfer it to the other phase and recharge it there. The image energy arises from a polarization of the membrane which can mathematically be described by image charges located a certain distance away from the interface in the region of the membrane. The dipole term accounts for the contribution of the dipole moments of lipid headgroups to the overall surface potential. As this interaction is hard to describe due to the discreteness of surface charge and variations in the dielectric constant near the surface, only a first order approximation can be made. Similar considerations hold for the neutral energy which is (apart from electrical contributions) the energy of transfer for a hydrophobic ion from one phase to the other including van der Waals, hydrophobic and steric factors.

\footnotetext{
${ }^{4} 500 \mathrm{~m}^{2}$ calculated for $1 \mathrm{~g}$ lipid solution $\left(\mathrm{MW} \approx 800 \mathrm{u}\right.$ ) assuming a lipid area of $0.71 \mathrm{~nm}^{2}$

${ }^{5}$ which equals the enthalpy of transfer, $\Delta H$
} 


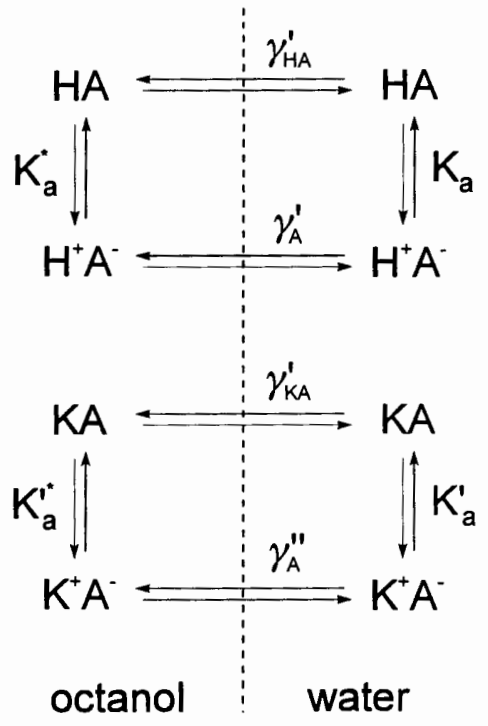

Figure 17: Reactions at the octanol/water interface involving $\mathrm{TeCP}$ in its neutral $(\mathrm{HA})$ and ionized $\left(\mathrm{A}^{-}\right)$ forms, with proton $\left(\mathrm{H}^{+}\right)$or potassium $\left(\mathrm{K}^{+}\right)$counterions.

The total energy according to this model exhibits a potential well for positively as well as for negatively charged ions close to the membrane/water interface. Therefore the negative surface charge does not hinder partitioning of anions into the membrane. This model sucessfully predicts experimental values for $\Delta G_{0}$ for the binding of tetraphenylphosphonium $\left(\mathrm{TPP}^{+}\right)$and tetraphenylboron $\left(\mathrm{TPB}^{-}\right)$(Flewelling and Hubbell, 1986).

A similar model might be applicable to the partitioning of ions into octanol by neglecting the energy terms related to surface interactions which are the dipole and image energies. Another important difference is the fact that electroneutrality of the phases needs to be satisfied. Therefore partitioning of $\mathrm{A}^{-}$into the octanol phase implicates the simultaneous partitioning of counterions. This co-partitioning of counterions is the limiting factor for $\gamma_{\mathrm{A}}$ and is the origin of the strong dependence on the ionic strength of the solution (Westall et al., 1985). Westall suggested the existence of free ions in the octanol phase on the basis of Bjerrum's model (Harned and Owen, 1958).

Figure 17 shows possible reactions at the octanol/water interface involving HA 
and $\mathrm{A}^{-}$. The individual partition coefficients are the sums of the partition coefficients for $\mathrm{A}^{-}$implying simultaneous partitioning of $\mathrm{H}^{+}$or $\mathrm{K}^{+}$.

$$
\gamma_{\mathrm{HA}}=\gamma_{\mathrm{HA}}+\gamma_{\mathrm{KA}} \quad \text { and } \quad \gamma_{\mathrm{A}}=\gamma_{\mathrm{A}}^{\prime}+\gamma_{\mathrm{A}}^{\prime \prime} \text {. }
$$

Recent computer simulations of ion transport across a 1,2-dichloroethane/water interface (Benjamin, 1993) show that the dynamics of the interface on a picosecond scale plays an important role in the transfer of $\mathrm{Cl}^{-}$ions.

\subsection{Distribution Ratio and Partition Coefficients}

Adsorption of charged molecules to biological membranes can adequately be modelled by the Gouy-Chapman theory for the diffuse double layer including a Langmuir adsorption isotherm, which has been described in detail in Section 2.4. From this model the linear partition coefficient, $\beta_{\mathrm{A}}^{m}$, can be obtained

$$
\beta_{\mathrm{x}}^{m}=\frac{(\mathrm{X})_{m}}{[\mathrm{X}]_{a q}} .
$$

The relation between the linear partition coefficient and the bulk partition coefficent, $\gamma_{\mathrm{x}}^{m}$, is given by

$$
\begin{aligned}
\gamma_{\mathrm{x}}^{m}= & \frac{\left\langle[\mathrm{X}]_{m}\right\rangle}{[\mathrm{X}]_{a q}} \quad \text { with } \\
\left\langle[\mathrm{X}]_{m}\right\rangle & =\frac{\int_{0}^{t}[\mathrm{X}(z)]_{m} \cdot d z}{t}
\end{aligned}
$$

where $[\mathrm{X}(z)]_{m}$ denotes the local concentration of the compound inside the membrane at a distance $\mathrm{z}$ from the surface (Smejtek and Wang, 1993).

The theoretical description for partitioning of compounds (charged or uncharged) between bulk phases is based on simple chemical equilibrium equations employing partition coefficients. For the determination of individual partition coefficients for TeCP and its ionized form, $\gamma_{\mathrm{HA}}=\frac{[\mathrm{HA}]_{Q}}{[\mathrm{HA}]_{W}}$ and $\gamma_{\mathrm{A}}=\frac{\left[\mathrm{A}^{-}\right]_{Q}}{\left[\mathrm{~A}^{-}\right]_{W}}$ respectively, a relationship 
between these parameters and a measurable quantity needs to be developed. This quantity is the distribution ratio, $D$, defined by

$$
D=\frac{[\mathrm{HA}]_{O}+\left[\mathrm{A}^{-}\right]_{O}}{[\mathrm{HA}]_{W}+\left[\mathrm{A}^{-}\right]_{W}}
$$

which can be obtained experimentally. The $p K_{a}$ of the compound in water is given by

$$
p K_{a}=-\log \frac{\left[\mathrm{A}^{-}\right]_{W}\left[\mathrm{H}^{+}\right]}{[\mathrm{HA}]_{W}}
$$

which yields together with $p H=-\log \left[\mathrm{H}^{+}\right]$

$$
\left[\mathrm{A}^{-}\right]_{W}=[\mathrm{HA}]_{W} 10^{p H-p K_{a}} .
$$

Substituting Eq. 44 into Eq. 42 results in

$$
D=\frac{\gamma_{\mathrm{HA}}+\gamma_{\mathrm{A}} 10^{p H-p K_{a}}}{1+10^{p H-p K_{a}}} .
$$

This equation relates $\gamma_{\mathrm{HA}}$ and $\gamma_{\mathrm{A}}$ to $D$ and $p H$ as parameters which can be directly obtained from experimental data. A nonlinear fit of value pairs $D$ and $p H$ to Eq. 45 yields $\gamma_{\mathrm{HA}}$ and $\gamma_{\mathrm{A}}$.

\subsection{Absorption Spectroscopy}

It was assumed that $\mathrm{TeCP}(\mathrm{HA})$ and its ionized form $\mathrm{A}^{-}$obey Beer's Law which relates the concentration of a substance to the intensity $I(x)$ of a beam of light traveling through a solution containing the substance. This can be expressed by

$$
I(x)=I_{0} e^{-A} \quad \text { with } \quad A=\epsilon x c
$$

where $I_{0}$ is the incident light intensity, $A$ the absorbance, $x$ the path length the light has traveled through the solution, $\mathrm{c}$ the concentration and $\epsilon$ the extinction coefficient of the compound in the specific solvent.

The mechanism behind this law is based on quantum physics. Molecules have 
(a)
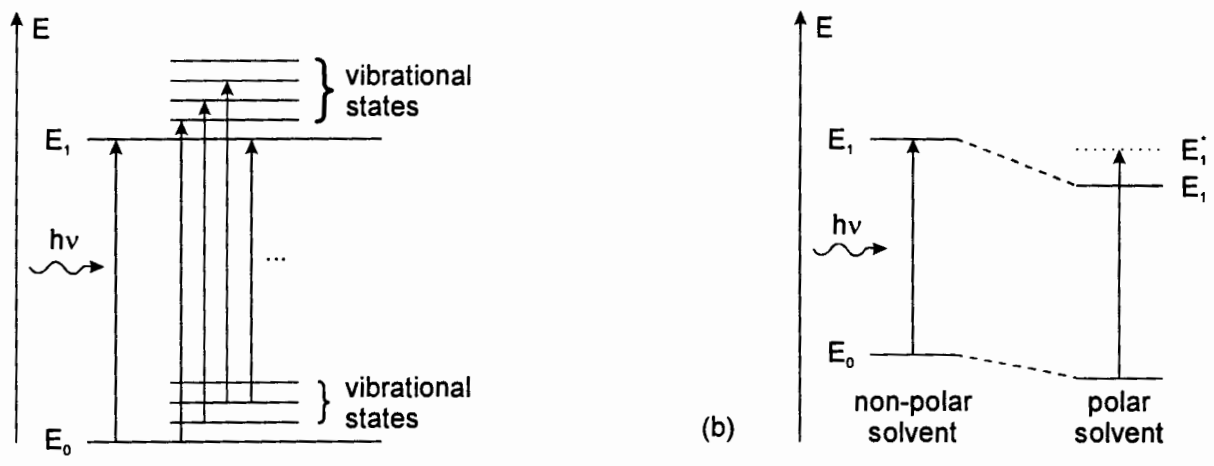

Figure 18: (a) Electronic and vibrational energy states of a molecule, $\mathrm{E}_{0}$ : ground state, $E_{1}$ : excited state; (b) solvent shift of energy transition (bathochromic shift), $\mathrm{E}_{1}^{*}$ : temporary excited state before reordering of solvent molecules

different energy states which are of electronic, rotational or vibrational origin. By absorbing any form of energy (e.g., kinetic energy or light) a molecule can undergo a transition from its ground state to an excited state. The energy $E$ of a photon is related to its wavelength $\lambda$ by

$$
E=\frac{h c_{m}}{\lambda},
$$

where $h$ is Planck's constant and $c_{m}$ the velocity of light in the medium. If a photon with an energy equal to the energy of a possible transition ${ }^{6}$ hits a molecule, the molecule undergoes a transition to an excited state by absorbing the photon. This results in a decrease of photon flux through the sample at that particular wavelength. Eq. 46 reflects this effect in the form of a classical relationship where the extinction coefficient incorporates the quantum mechanical properties of the specific molecule.

The energy gap between two electronic states is usually of the order of an electron volt $(\mathrm{eV})$ which corresponds to the energy of visible or ultraviolet (UV) light. Rotational and vibrational energy gaps are much smaller and can therefore be excited by infrared (IR) light or microwave radiation. As the energy of these transitions is relatively low, some molecules are in vibrational or rotational excited states at room

\footnotetext{
${ }^{6}$ the transitions are restricted by the quantum mechanical selection rules
} 
temperature due to thermal excitation. This results in a splitting of the electronic energy states as vibrational levels are superimposed (see Figure 18a). Molecules can therefore undergo electronic and vibrational/rotational transitions simultaneously which manifests itself as a broadening of the absorption peak.

Changes in the energetic behavior of the molecule like conformational changes, group substitutions, solvent interactions, etc., alter the extinction coefficient. Of particular importance in our case are solvent effects as we use buffer (water) and octanol as solvents in the experiment. A shift in the absorbance wavelength can be caused by the properties of the solvent. Most molecules are more polar in the excited state than in the ground state. This leads to a different ordering of the polar water molecules around the solute molecule, depending on its state. The water molecules cannot rearrange during the very short transition process $(\approx 1 \mathrm{fs})$ from the ground to the excited state which results in a temporary energy rise of the excited state. Therefore a higher excitation energy is needed which manifests itself in a shorter wavelength of the absorbed light (see Figure 18b). This effect is the origin for the shift in the absorbance wavelength for the neutral form of TeCP from $\lambda=298 \mathrm{~nm}$ in water (as a polar solvent) to $\lambda=301.5 \mathrm{~nm}$ in octanol (as a nonpolar solvent). 


\section{Chapter 6}

\section{Materials and Methods}

\subsection{Chemicals}

Potassium chloride was bought from EM Science, Cherry Hill, NJ. Boric acid and potassium citrate were both from Matheson, Coleman \& Bell Manufacturing Chemists, Norwood, Ohio. 2,3,4,5-tetrachlorophenol (98\% pure) and 1-octanol (spectroscopic grade) were obtained from Aldrich Chemical Company, Milwaukee, WI. Chemicals were used without further purification. All solutions were prepared with deionized water.

\subsection{Spectrophotometric Measurements}

The spectrophotometer used in all experiments was Model DU-7 from Beckman Instruments, Irvine, CA. For the buffer phase $10 \mathrm{~mm}$ (Pyrocell Manufacturing Co., Inc., Westwood, NJ), $50 \mathrm{~mm}$ and $1 \mathrm{~mm}$ cuvettes (Spectrocell Corp., Orland, PA) were used. The octanol phase was measured in $10 \mathrm{~mm}$ cuvettes from Spectrocell. According to the specifications the spectrophotometer has $\pm 0.5 \mathrm{~nm}$ wavelength accuracy, $\pm 0.5 \%$ photometric accuracy and a baseline drift below $0.003 \mathrm{~A} / \mathrm{hr}$. A tungsten lamp is employed for the visible region $(700-315 \mathrm{~nm})$ and a deuterium 


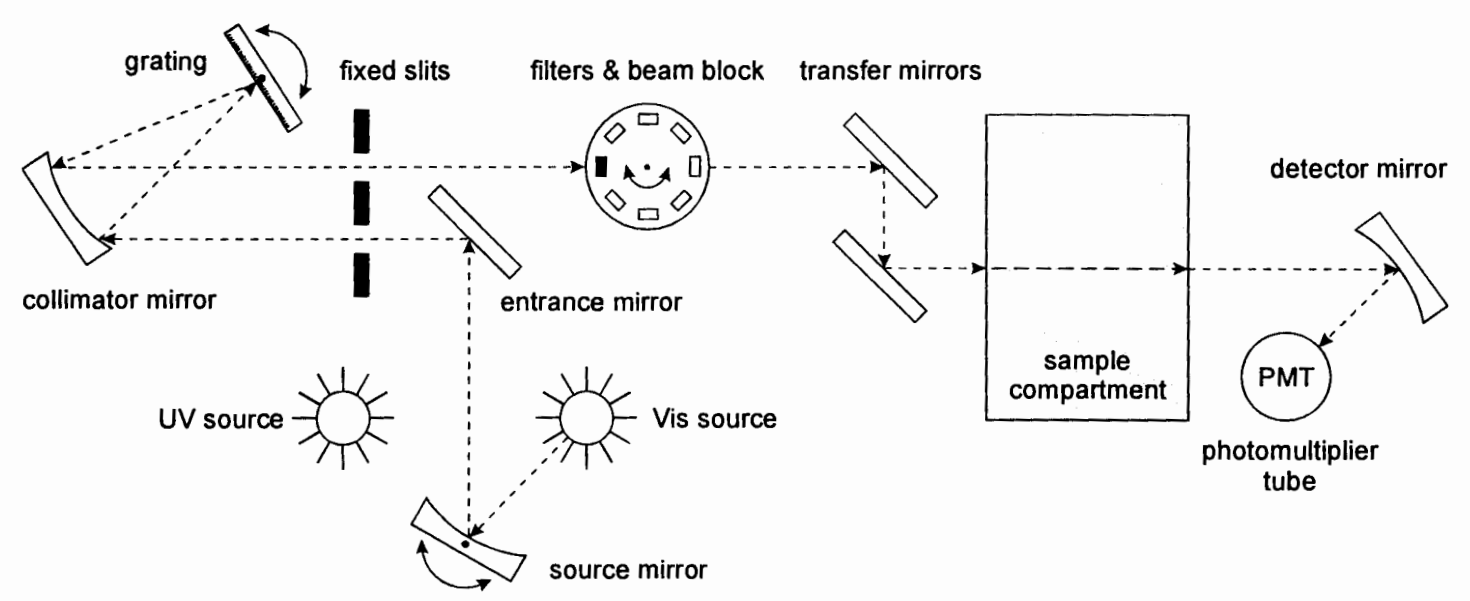

Figure 19: Block diagram of the optical path in the spectrophotometer (Beckman, 1982).

lamp for the UV region (315-200 nm). A microprocessor controlled mirror selects the lamp for the wavelength region being scanned. The offset at the $315 \mathrm{~nm}$ switching point was repeatedly checked to be within specifications. The light beam is narrowed by the slit assembly and focussed on the grating by the collimator mirror as shown in Figure 19. The grating breaks the light beam into its spectrum. It is controlled by a stepper motor to adjust the wavelength being reflected back onto the collimator mirror. From here the light is focussed through a set of microprocessorcontrolled blocking filters which provide constant energy throughout the spectrum. Two transfer mirrors position the beam path so that it can pass through the sample compartment. The detector mirror focusses the beam onto the photomultiplier tube which produces an electrical signal that is amplified and then analysed by a microprocessor.

A scan speed of $600 \mathrm{~nm} / \mathrm{min}$ at a resolution of $0.5 \mathrm{~nm}$ was used throughout the experiment. The spectrophotometer was controlled by an IBM compatible PC running a program designed for that purpose. The software is described in Appendix C. 
To perform a measurement, a background solution and a sample solution is needed. The background has the same consistency as the sample only without the compound to be measured. Therefore, two cuvettes are needed which were rinsed with methanol and acetone, air dried and carefully cleaned with lens tissue before each experiment. The absorbance difference between both cuvettes ${ }^{1}$ was not allowed to exceed 0.002 A. During the experiment the cuvettes were rinsed at least three times with the new solutions before a measurement was performed.

\subsection{Phase Preparation}

In all experiments citrate/borate/phosphate buffer $(2 \mathrm{mM} / 2 \mathrm{mM} / 0.5 \mathrm{mM}), \mathrm{B}^{-3}$, with a salt concentration of $0.03 \mathrm{M} \mathrm{KCl}$ was used as the aqueous phase. The addition of the salt was neccessary to provide the same experimental conditions as in the membrane adsorption measurements (Blochel, 1992) and to maintain a constant $\mathrm{K}^{+}$concentration ${ }^{2}$ as the partitioning depends strongly on this parameter (Westall et al., 1985). Buffer solutions were prepared by diluting a $\mathrm{B}^{-1}$ stock solution and adding the neccessary volume of $\mathrm{a} \approx 2 \mathrm{M} \mathrm{KCl}$ solution.

To reduce the effects ${ }^{3}$ of water solvation in octanol and vice versa, ${ }^{4}$ presaturated phases have been used for all experiments. Octanol and buffer solutions were combined at a ratio $1: 5$ in $250 \mathrm{~mL}$ polypropylene centrifuge bottles. The bottles were shaken for one hour on a Wrist-Action shaker model BB from Burell Corp., Pittsburgh, PA, and then centrifuged at $1500 \mathrm{~g}$ in a IEC DPR-6000 centrifuge.

Extreme care has to be taken when separating the two phases. The octanol phase was removed from the top of the aqueous phase with a syringe. The syringe was fixed on a holder to facilitate one-hand operation. An octanol layer of approximately 3

\footnotetext{
${ }^{1}$ the cuvettes were filled with the respective background since measurements with dry cuvettes gave false results

${ }^{2}$ the addition of $\mathrm{KCl}$ and $\mathrm{KOH}$ during titration is negligible

${ }^{3}$ change in volume and spectroscopic properties

${ }^{4}$ water has a high solubility $(2.3 \mathrm{M})$ in octanol, whereas octanol is poorly soluble in water (4.5 $\mathrm{mM})$
} 

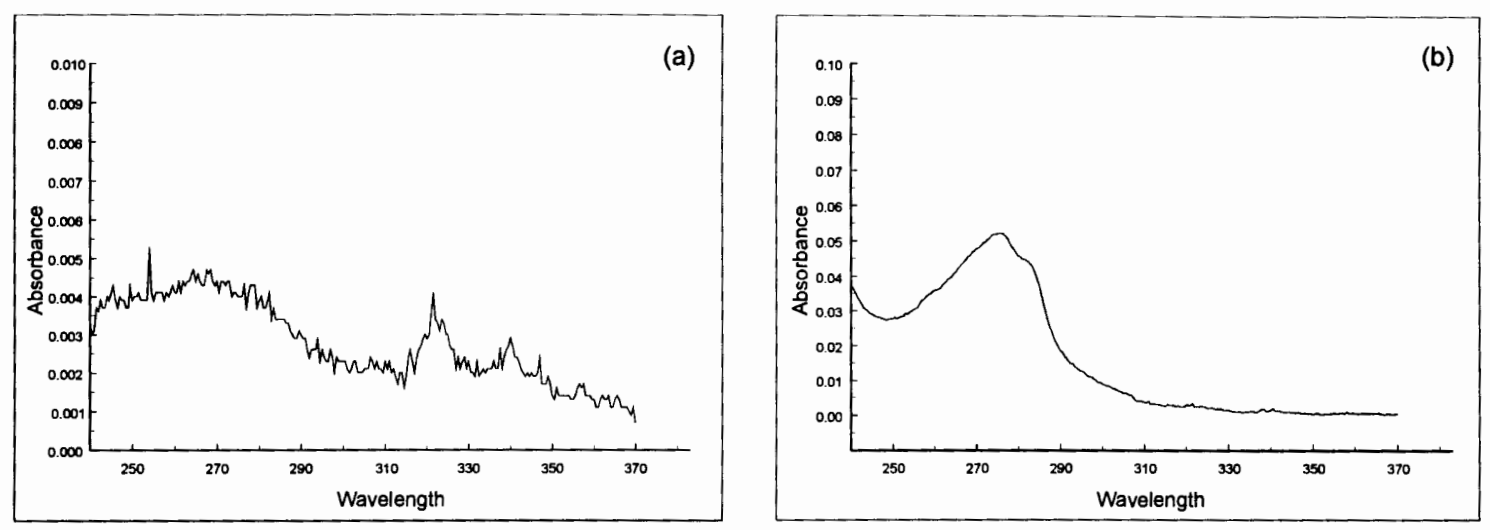

Figure 20: (a) Spectrum of the combined aqueous phase used for the partitioning experiment after saturation with octanol, measured against pure buffer; (b) Spectrum of the combined octanol phase used for the partitioning experiment after saturation with buffer, measured against pure octanol.

$\mathrm{mm}$ on top of the aqueous phase was always discarded to eliminate interfacial effects of the mixing process. The aqueous phase was moved to a stock container with a $150 \mathrm{~mL}$ pipet, again discarding the last $5 \mathrm{~mm}$. For the partitioning experiment the phases of two saturation batches consisting of six bottles each were combined.

The "quality" of saturation was examined spectrophotometrically using unsaturated solutions as background. The difference in absorbance for the saturated buffer phase in the region of interest $(300-330 \mathrm{~nm}$ ) had to be below $0.005 \mathrm{~A}$ (see Figure 20a). The saturated octanol phase exhibited a peak at around $275 \mathrm{~nm}$ whose origin is unknown (see Figure 20b). During several saturation batches the main features of the spectrum were extracted and served as criteria for the success of the saturation.

The formation of octanol micelles in the aqueous phase and vice versa is very likely to occur as it is reported for various oil/water emulsions (Cazabat and Langevin, 1980; Smit et al., 1990). The size of these micelles is of the order of a wavelength $(\approx 200 \mathrm{~nm})$ and therefor causes scattering of light in the UV region. This results in an increase in the measured absorbance and might be the reason for the peak at 
$275 \mathrm{~nm}$ in saturated octanol. The possible effects of micelle or emulsion formation has been neglected in this treatment.

\subsection{Extinction Coefficients}

Exctinction coefficients for TeCP in both the aqueous and the octanol phase were needed for the interpretation of the partitioning data. For the aqueous phase only the extinction coefficient for the ionized form was determined as all samples in the partitioning experiment were titrated to a high $p H$ for the spectroscopic determination of TeCP concentration.

TeCP sample solutions for the aqueous phase were prepared from stock solutions ranging between $1 \mathrm{mM}$ and $5 \mathrm{mM}$ due to the limited solubility of TeCP (see Table 3). To increase the solubility of $\mathrm{TeCP}, \mathrm{KOH}$ was added in small amounts for the preparation of the $5 \mathrm{mM}$ stock solution. It was not possible to dissolve TeCP in saturated buffer as aggregates of $\mathrm{TeCP}$ and octanol seem to form. All stock solutions were therefore prepared from unsaturated buffer but diluted with saturated one. The aqueous solution was titrated to a $p H$ well above the $p K_{a}$ of 6.35 (Schellenberg et al., 1984). As TeCP has a high solubility in octanol, the stock solutions had concentrations of $5 \mathrm{mM}$ and $17 \mathrm{mM}$. It was found that the extinction coefficient for the saturated and the pure solutions are the same within our measurement accuracy. Sample concentrations ranged from $0.1 \mathrm{mM}$ to $0.4 \mathrm{mM}$ for both solvents.

\subsection{Partitioning}

The samples for the partitioning experiment were prepared from a $20.0 \mathrm{mM}$ stock solution of TeCP dissolved in saturated octanol and $p H$-adjusted saturated buffer. We used $25 \mathrm{~mL}$ of buffer and $5 \mathrm{~mL}$ of octanol keeping the octanol:water ratio 1:5, which was the same as used in the phase-saturation procedure. The initial $p H$ of the buffer phase ranged between 3.23 and 12.3 resulting in a final $p H$ between 3.42 and 
11.9 measured with a Model 6072 microcomputer $p H$ meter from Jenco Electronics, Taipei, Taiwan.

The experiment was performed using the same preparation methods as described for the presaturation of the phases. For each $p H$ value two $50 \mathrm{~mL}$ glass centrifuge tubes were prepared, one containing octanol with $\mathrm{TeCP}$ and the other just presaturated octanol which was used as background in the spectrophotometic measurements. Attention has to be paid to the position of the tubes on the shaker to assure the mixing of the whole solution. Centrifuging at $1500 \mathrm{~g}$ was done in a Safeguard centrifuge from Clay-Adams Inc., New York, NY.

Samples were always taken from the aqueous phase. In three cases samples were also taken from the octanol phase to determine the mass balance of $\mathrm{TeCP}$ which was found to be well within our error limits. At high final $p H$, dilution of the sample ${ }^{5}$ was neccessary as the concentration of $\mathrm{TeCP}$ in the buffer phase exceeded the absorbance limit of the instrument. Low values of the final $p H$ resulted in concentrations of the order of the detection limit of the spectrophotometer. For the lowest $p H$ a cuvette with a path length of $5 \mathrm{~cm}$ was used.

The $p H$ of the sample from the aqueous phase was measured immediately after the sample was taken to prevent a drop in $p H$ caused by dissolution of $\mathrm{CO}_{2}$ from air. Samples with a final $p H$ lower than 9.4 were titrated to a $p H$ well above the $p K_{a}$ to transform the major part of TeCP into its ionized form. This procedure was chosen since only information about the total TeCP concentration was needed, rather than the concentration of the neutral and the ionized species separately.

\footnotetext{
${ }^{5}$ alternatively a cuvette with $1 \mathrm{~mm}$ path length was used instead of $1 \mathrm{~cm}$
} 


\section{Chapter 7}

\section{Results and Discussion}

\subsection{Extinction Coefficients}

The extinction coefficient for 2,3,4,5-tetrachlorophenol in buffer and octanol has been measured for several concentrations using three different stock solutions. Table 1 lists the sample concentrations and the obtained values for the extinction coefficients. All concentration determinations for the partitioning experiment are based
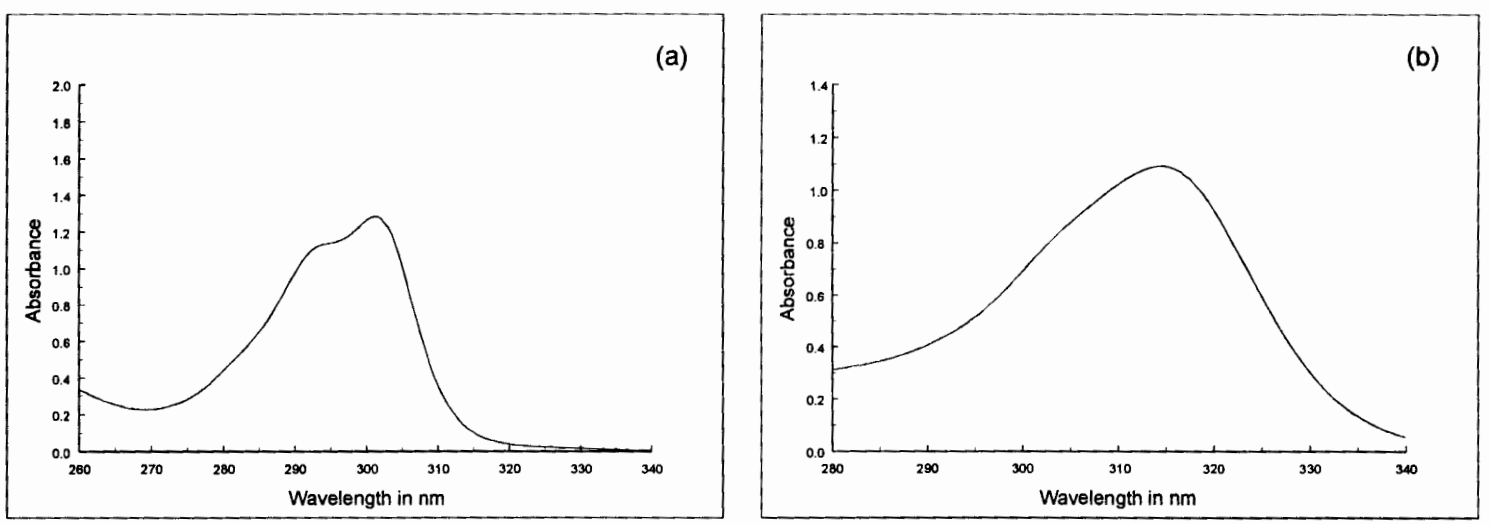

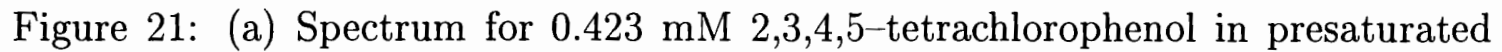

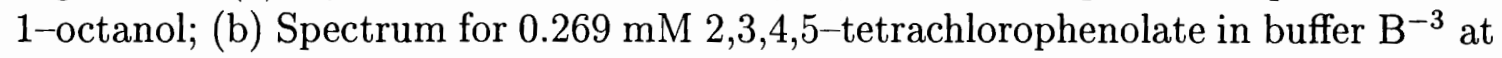
pH 10.8 . 


\begin{tabular}{|c|c|c|c|c|}
\hline solution & $\begin{array}{c}\text { stock conc. } \\
{[\mathrm{mM}]}\end{array}$ & $\begin{array}{l}\text { sample conc. } \\
{[\mathrm{mM}]}\end{array}$ & $\begin{array}{l}\text { ext. coeff. } \\
{\left[\frac{1}{\mathrm{M} \cdot \mathrm{cm}}\right]}\end{array}$ & $\begin{array}{l}\text { average } \\
{\left[\frac{1}{\mathrm{M} \cdot \mathrm{cm}}\right]}\end{array}$ \\
\hline \multirow{2}{*}{$\mathrm{B}^{-3}+\mathrm{KOH}$} & \multirow{2}{*}{5.00} & 0.200 & 4400 & \multirow{6}{*}{$\begin{array}{c}4460 \pm 40 \\
(\lambda=314.5 \mathrm{~nm})\end{array}$} \\
\hline & & 0.100 & 4450 & \\
\hline \multirow{2}{*}{$B^{-3}$} & \multirow{2}{*}{1.06} & 0.264 & 4500 & \\
\hline & & 0.265 & 4470 & \\
\hline \multirow{2}{*}{$\mathrm{B}^{-3^{*}}$} & \multirow{2}{*}{1.09} & 0.107 & 4460 & \\
\hline & & 0.298 & 4430 & \\
\hline \multirow{4}{*}{ Octanol } & \multirow{4}{*}{5.48} & 0.329 & 3000 & \multirow{8}{*}{$\begin{array}{c}3020 \pm 60 \\
(\lambda=301.5 \mathrm{~nm})\end{array}$} \\
\hline & & 0.329 & 2940 & \\
\hline & & 0.439 & 3000 & \\
\hline & & 0.110 & 3080 & \\
\hline \multirow{2}{*}{ Octanol $^{*}$} & \multirow{2}{*}{5.28} & 0.105 & 3120 & \\
\hline & & 0.423 & 3040 & \\
\hline \multirow{2}{*}{ Octanol ${ }^{*}$} & \multirow{2}{*}{17.2} & 0.345 & 2980 & \\
\hline & & 0.207 & 3030 & \\
\hline
\end{tabular}

Table 1: Extinction coefficients for $\mathrm{TeCP}$ in buffer and in octanol; the star "**" marks presaturated phases.

on the average values of $\epsilon=4460 \pm 40(\lambda=314.5 \mathrm{~nm})$ for the buffer phase and $\epsilon=3020 \pm 60(\lambda=301.5 \mathrm{~nm})$ for the octanol phase.

\subsection{Partitioning}

The partitioning experiment was performed for 15 values of the initial $p H$ of the buffer phase. Table 2 shows a summary of the obtained data. The distribution ratio Eq. 42 was calculated from the spectrophotometrically obtained aqueous concentrations of TeCP applying the mass--balance equation for the system.

Figure 22 shows the distribution ratio between octanol and buffer for the measured $p H$ values. Solid curves illustrate the distribution ratio predicted from Eq. 


\begin{tabular}{|c|c|c|c|c|}
\hline initial $p H$ & final $p H$ & $\begin{array}{c}{[\mathrm{HA}]_{W}+\left[\mathrm{A}^{-}\right]_{W}} \\
{[\mathrm{mM}]}\end{array}$ & $\begin{array}{c}{[\mathrm{HA}]_{O}+\left[\mathrm{A}^{-}\right]_{O}} \\
{[\mathrm{mM}]}\end{array}$ & $\log D$ \\
\hline \hline 12.3 & 11.9 & 2.05 & 9.77 & 0.689 \\
\hline 11.8 & 11.0 & 1.79 & 11.1 & 0.792 \\
\hline 11.4 & 10.0 & 0.678 & 16.6 & 1.39 \\
\hline 11.4 & 10.0 & 0.872 & 15.6 & 1.25 \\
\hline 11.2 & 9.76 & 0.678 & 16.6 & 1.39 \\
\hline 10.7 & 9.40 & 0.360 & 18.2 & 1.70 \\
\hline 10.4 & 9.35 & 0.295 & 18.5 & 1.80 \\
\hline 10.8 & 9.14 & 0.231 & 18.8 & 1.91 \\
\hline 9.99 & 9.07 & 0.182 & 19.1 & 2.02 \\
\hline 9.00 & 8.41 & 0.0710 & 19.7 & 2.44 \\
\hline 7.77 & 7.81 & 0.0215 & 19.9 & 2.97 \\
\hline 7.47 & 7.48 & 0.0107 & 20.0 & 3.27 \\
\hline 6.94 & 7.10 & 0.0052 & 20.0 & 3.58 \\
\hline 6.40 & 6.62 & 0.0025 & 20.0 & 3.90 \\
\hline 3.23 & 3.42 & 0.0012 & 20.0 & 4.22 \\
\hline
\end{tabular}

Table 2: Experimental data from octanol/water partitioning of TeCP. 


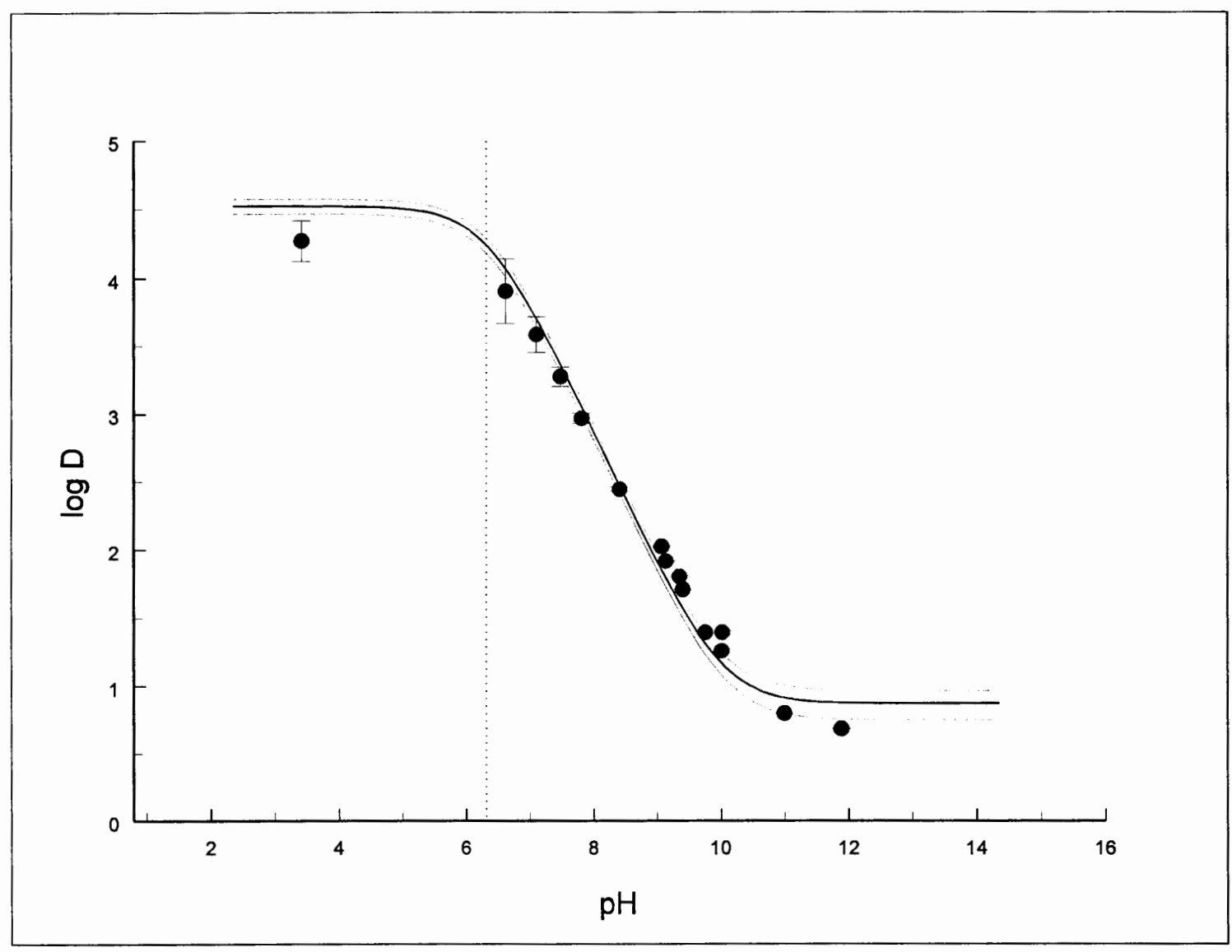

Figure 22: $p H$-dependence of the distribution ratio of TeCP in octanol and water; gray lines are the standard deviation, the dotted line marks the $p K_{a}$ 


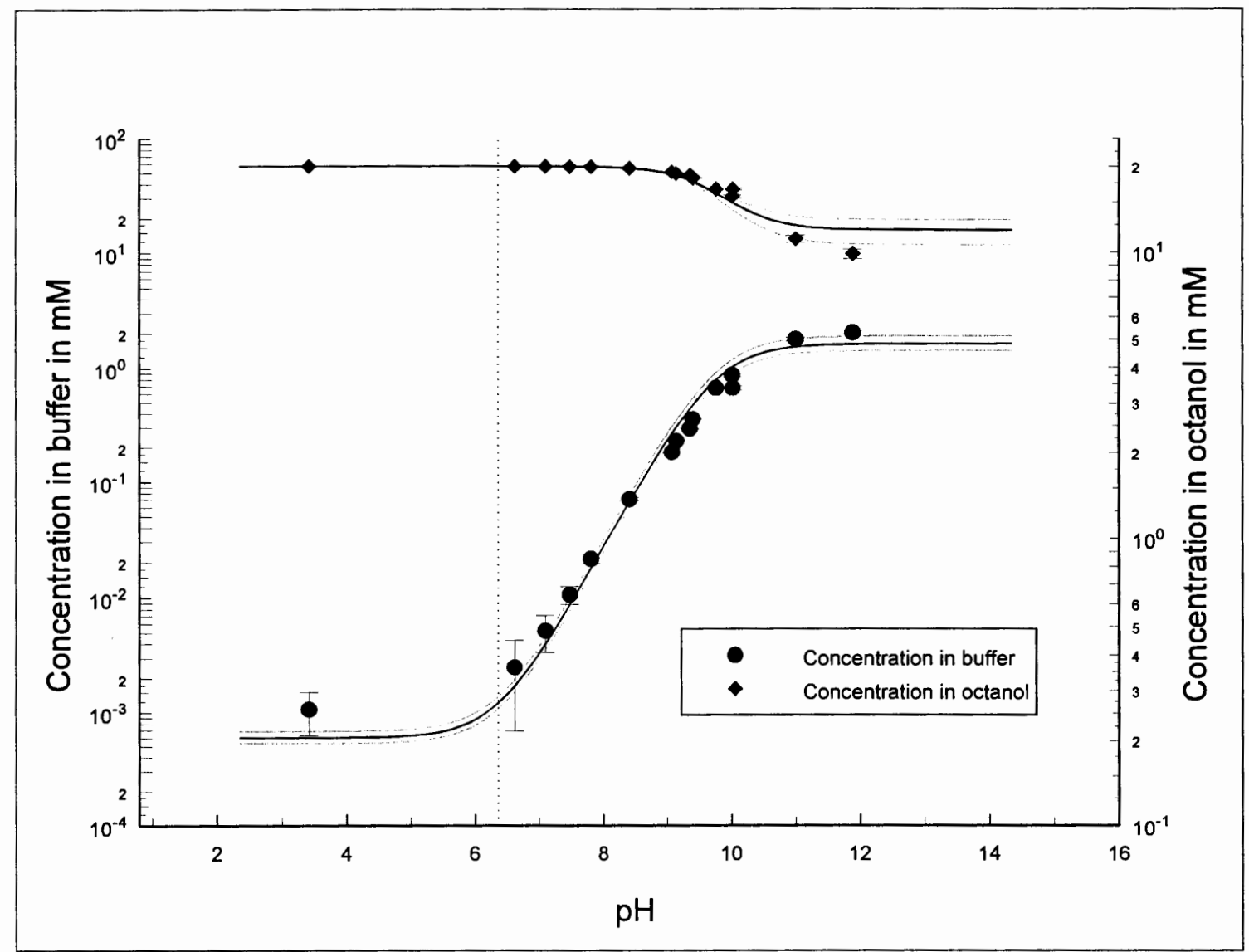

Figure 23: $p H$-dependence of the concentration of TeCP in octanol and water; gray lines are the standard deviation, the dotted line marks the $p K_{a}$ 
45 employing $\gamma_{\mathrm{A}}=7.4 \pm 1.8$ and $\gamma_{\mathrm{HA}}=33000 \pm 4000$. These values were a nonlinear least square fit of the experimental data to Eq. 45. Gray lines show the standard deviation of the predicted distribution ratio. As expected for very high and very low values of the $p H$, the distribution ratio saturates and corresponds to that for the neutral and ionized TeCP, $\gamma_{\mathrm{HA}}$ and $\gamma_{\mathrm{A}}$ respectively. The value obtained for $\log \gamma_{\mathrm{HA}}=4.52 \pm 0.05$ is in good agreement with the literature values that are ranging from $\log P_{\text {ow }}=4.21$ to 4.87 , depending on the method used (Veith and Mekenyan, 1993; Bryant and Schultz, 1994; Schellenberg et al., 1984).

The experiment was designed to produce a strong $p H$ dependence of the concentration profiles (Figure 23). Volume ratio and initial TeCP concentration were chosen to get a maximum concentration variation in the aqueous phase utilizing the widest range of measurable absorbances. The high aqueous concentration of TeCP for $p H-p K_{a}>2$ reflects upon the value of the octanol/water partition coefficient of the ionized species.

\subsection{Conclusions}

The knowledge of the partition coefficient for the negatively charged species of 2,3,4,5-tetrachlorophenolate, $\gamma_{\mathrm{A}}$, makes it possible to compare it with the value for the linear partition coefficient for adsorption to phospholipid membrane, $\beta_{\mathrm{A}}^{m}$ (by means of Eq. 40). Assuming a membrane thickness of $t=38 \AA$ (McIntosh and Simon, 1986) one obtains a membrane bulk partition coefficient $\gamma_{\mathrm{A}}^{m}=3000$. This value is 400 times greater than that predicted by the octanol/water partition coefficient. Smejtek and Wang (1993) found analogous behavior for PCP using similar experimental methods. A summary of relevant properties and results is given in Table 3.

The major result of this study is that the octanol/water partition coefficient is not a suitable means for the prediction of distribution of the ionized form of 


\begin{tabular}{|c|c|c|c|}
\hline MW (u) & $p K_{a}$ & $C^{S}(\mathrm{mg} / \mathrm{L})$ & $\log P_{\text {OW }}$ \\
\hline 231.89 & $6.35 \pm 0.02^{a}$ & 67.4 & $4.87 \pm 0.08^{a}$ \\
\hline \hline $\log \beta_{\mathrm{A}}^{m}$ & $\log \gamma_{\mathrm{A}}^{m}$ & $\log \gamma_{\mathrm{A}}$ & $\log \gamma_{\mathrm{HA}}$ \\
\hline$-5.246 \pm 0.015^{b}$ & $3.475 \pm 0.015^{b}$ & $0.87 \pm 0.10$ & $4.52 \pm 0.05$ \\
\hline
\end{tabular}

Table 3: Selected properties of 2,3,4,5-tetrachlorophenol.

\footnotetext{
${ }^{a}$ Schellenberg (1984)
}

${ }^{b}$ Blochel (1992)

2,3,4,5-tetrachlorophenol between water and lipid membranes and that $P_{\text {ow }}$ is expected to seriously underestimate the concentration of ionized TeCP in biological cell membranes.

The rationale for the difference can be based on the physical properties of the two model systems. Octanol, as a bulk phase, requires maintainance of electroneutrality and therefore co-partitioning of counterions which requires additional free energy. In contrast $\mathrm{TeCP}$ ions in the lipid membrane are expected to be stabilized by a deep potential energy minimum close to the lipid/water interface (Flewelling and Hubbell, 1986). These ions are effectively screened by the counterions from the aqueous phase. This interaction is expected to lower the electrostatic energy of the membrane system compared to that in bulk octanol phase. 


\section{Appendix A}

\section{Derivations}

\section{A.1 Gouy-Chapman Theory}

Consider an infinite uniformly charged surface in contact with an infinite homogeneous electrolyte. Ions in an electric field obey a Boltzmann distribution Eq. 1 which in return gives rise to a potential originating from the spatial density

$$
\rho(x)=\sum_{i} z_{i} e N_{i}(x)
$$

Combining this equation with Poisson's equation, Eq. 2, yields the Poisson-Boltzmann equation

$$
\frac{d^{2} \psi(x)}{d x^{2}}=-\frac{1}{\epsilon_{r} \epsilon_{0}} \sum_{i} z_{i} e N_{i}(\infty) \exp \left(-\frac{z_{i} e \psi(x)}{k T}\right)
$$

which can be integrated using the boundary conditions

$$
\lim _{x \rightarrow \infty} \psi(x)=0 \quad \text { and } \quad \lim _{x \rightarrow \infty} x \frac{d \psi(x)}{d x}=0
$$

and the identity

$$
2 \frac{d^{2} \psi(x)}{d x^{2}}=\frac{d}{d x}\left(\frac{d \psi(x)}{d x}\right)^{2}
$$

One obtains

$$
\left(\frac{d \psi(x)}{d x}\right)^{2}=\frac{2 k T}{\epsilon_{r} \epsilon_{0}} \sum_{i} N_{i}(\infty)\left[\exp \left(-\frac{z_{i} e \psi(x)}{k T}\right)-1\right]
$$


Applying the electroneutrality condition ${ }^{1}$ to the double layer gives

$$
\sigma_{m}=-\int_{0}^{\infty} \rho(z) d z
$$

Substituting Eqs. 2 and 52 into Eq. 53 gives the Gouy-Chapman equation, Eq. 4.

$$
\begin{aligned}
\sigma_{m} & =\int_{0}^{\infty} \epsilon_{r} \epsilon_{0} \frac{d^{2} \psi(x)}{d x^{2}} d x=-\epsilon_{r} \epsilon_{0}\left(\frac{d \psi(x)}{d x}\right)_{x=0} \\
& =2 \epsilon_{r} \epsilon_{0} k T \sum_{i} N_{i}(\infty)\left[\exp \left(-\frac{z_{i} e \psi(0)}{k T}\right)-1\right]^{\frac{1}{2}} .
\end{aligned}
$$

\section{A.2 Rod Model}

The charge density of RuR in this model is found by extending the Boltzmann distribution Eq. 1 to three charges type A, C at the ends and type B, at the center of a rod each with distributions $N_{A}, N_{C}$ and $N_{B}$. They account for the position of the respective charge and a spatial average over all possible positions of the other two charges which are restricted by the membrane. One obtains

$$
N_{A}(r)=N_{A}(\infty) \frac{\int_{0}^{2 \pi} d \phi \int_{0}^{\min \left[\pi, \cos ^{-1}(-r / a)\right]} d \theta \sin \theta g(r+0.5 a \cos \theta, \theta)}{\int_{0}^{2 \pi} d \phi \int_{0}^{\pi} d \theta \sin \theta}
$$

where

$$
g(r, \theta)=\exp \left(-\frac{2 e}{k T}[\psi(r)+\psi(r-0.5 a \cos \theta)+\psi(r+0.5 a \cos \theta)]\right)
$$

is the probability of finding a molecule ${ }^{2}$ at a distance $r$ from the surface making an angle $\theta$ with the normal (see Figure 7). The maximum angle of the molecule without penetrating the membrane is given by $\cos ^{-1}(-r / a)$. Substituting $z=a \cos \theta$ and Eq. 56 into Eq. 55 and performing the $d \phi$ integration one obtains

$$
N_{A}(r)=N_{A}(\infty) e^{-\frac{2 e \psi(r)}{k T}} \frac{1}{2 a} \int_{\max [-a,--r]}^{a} d z e^{-\frac{2 e}{k T}[\psi(r+z / 2)+\psi(r+z)]} .
$$

\footnotetext{
${ }^{1}$ the surface charge density $\sigma_{m}$ is balanced by the net charge in the aqueous diffuse double layer

${ }^{2}$ the position of the molecule is determined by the position of its center
} 
Similar considerations for charges type B yield a density of

$$
\begin{aligned}
N_{B}(x) & =N_{B}(\infty) \frac{\int_{0}^{2 \pi} d \phi \int_{\max \left[0, \cos ^{-1}(2 x / a)\right]}^{\min \left[\pi, \cos ^{-1}(-2 x / a)\right]} d \theta \sin \theta g(x, \theta)}{\int_{0}^{2 \pi} d \phi \int_{0}^{\pi} d \theta \sin \theta} \\
& =N_{B}(\infty) e^{-\frac{2 e \psi(x)}{k T}} \frac{1}{2 a} \int_{\max [-a,-2 x]}^{\min [a, 2 x]} d z e^{-\frac{2 e}{k T}[\psi(x-z / 2)+\psi(x+z / 2)]} .
\end{aligned}
$$

These densities together with Eq. 48 and the Poisson equation, Eq. 2, yield ${ }^{3}$

$$
\frac{d^{2} \psi(x)}{d x^{2}}=-\frac{\sum_{i} z_{i} e N_{i}}{\epsilon_{r} \epsilon_{0}}
$$

A first formal integration using the boundary conditions, Eq. 50, yields

$$
\frac{d \psi(x)}{d x}=\left.\frac{d \psi(x)}{d x}\right|_{x=0}-\frac{1}{\epsilon_{r} \epsilon_{0}} \int_{0}^{x} d y \sum_{i} z_{i} e N_{i}(y)
$$

Integrating by parts and considering the boundary conditions gives the identity

$$
\psi(x)=\psi(0)+x \frac{d \psi(x)}{d x}-\int_{0}^{x} d y(y) \frac{d^{2} \psi(y)}{d y^{2}}
$$

Substitution of Eqs. 59 and 60 into Eq. 61 yields

$$
\psi(x)=\psi(0)+\left.x \frac{d \psi(x)}{d x}\right|_{x=0}-\frac{1}{\epsilon_{r} \epsilon_{0}} \int_{0}^{x} d y(x-y) \sum_{i} z_{i} e N_{i}(y)
$$

which is identical to Eq. 11.

\footnotetext{
${ }^{3} z_{A}=z_{B}=z_{C}=2$ as $N_{A}, N_{C}$ and $N_{B}$ represent the densities of the 2e-charges at the ends and the center of the rod, respectively
} 


\section{Appendix B}

\section{Numerical Methods}

The algorithms for solving the equations were implemented in Visual Basic (VB) 3.0 professional edition for Windows ${ }^{\circledR}$. This software development tool was used as it provides a fast way to write programs due to its extended basic language set with dynamic memory management and interactive debugging features. The latter is extremely helpful as one can perform code changes while the program is running. The price for this advantage is a comparably slow performance due to the fact that the program is interpreted ${ }^{1}$ rather than compiled. It would be desirable to have both: an interpreter for program development and a compiler for the final executable file. Program development was done on a IBM compatible 486/33, the final calculations were performed on four IBM compatible Pentium ${ }^{\circledR} / 90$ systems. Computation times for the Rod Model (the most time intensive) ranged from 12 to 24 hours for the electrostatic part and between 2 and 3 days for the adsorption calculations. ${ }^{2}$ An estimated 10000 integrations have to be performed accumulating to about one billon floating point operations.

\footnotetext{
1 interpreted "p-code"

${ }^{2}$ due to the fact, that for each iteration step in the Adsorption Model the complete Rod Model has to be solved
} 


\section{B.1 Integration}

All numerical integrations were performed using a combination of Simpson's and the trapezoid rule (Davis and Rabinowitz, 1984). Simpson's rule in compound form assumes an even number of equally spaced subintervals $a=x_{0}<x_{1}<\ldots<x_{2 n-1}<$ $x_{2 n}=b$ with $x_{i+1}-x_{i}=h$. Using the notation $f_{i}=f\left(x_{i}\right)$, Simpson's rule can be written as

$\int_{x_{0}}^{x_{2 n}} f(x) d x=\frac{h}{3}\left[f_{0}+4\left(f_{1}+f_{3}+\cdots+f_{2 n-1}\right)+2\left(f_{2}+f_{4}+\cdots+f_{2 n-2}\right)+f_{2 n}\right]+E_{n}$, where $E_{n}$ is the upper limit of the error

$$
E_{n}=-\frac{(b-a)^{5}}{180 N^{4}} f^{(4)}(\zeta), \quad a<\zeta<b \quad \text { and } \quad N=2 n
$$

As one can see from the definition of $E_{n}$, Simpson's rule is exact for polynomials of degree less than 4 . The application of the method is restricted to $N \geq 2$ and to an even number of subintervals. For $N=1$ the trapezoid rule

$$
\int_{x_{0}}^{x_{1}} f(x) d x=\frac{h}{2}\left(f_{0}+f_{1}\right)
$$

is used. For an odd number of subintervals the integration is performed according to Simpson's rule without the last subinterval which is accounted for by an additional term calculated with the trapezoid rule.

Tests showed that a grid of at least 1000 intervals was required for the first four Debye lengths to keep the error well below $1 \%$. For reasonable computation times an interpolation for another four Debye lengths was performed assuming exponential decay, which was found to produce correct results within our error limits.

\section{B.2 Numerical Solution of the GC Model}

The Gouy-Chapman equation, Eq. 4, together with the electroneutrality condition, Eq. 3, must be solved in order to obtain $\psi(x)$. This was done using the formal 
solution of the Poisson equation, Eq. 11,

$$
\psi(x)=\psi(0)+\left.x \frac{d \psi(x)}{d x}\right|_{x=0}-\frac{1}{\epsilon_{r} \epsilon_{0}} \int_{0}^{x} d y(x-y) \sum_{i} z_{i} e N_{i}(y)
$$

which has been derived in Section A.2. For $N_{i}(y)$ the regular Boltzmann distribution, Eq. 1, was used. To perform the integration, $\psi(0)$ and $\left.\frac{d \psi(x)}{d x}\right|_{x=0}$ are needed. Eq. 4 is solved iteratively for $\psi(0)$ with a root-finding subroutine adopted from a Fortran-77 subroutine package using successive bisectioning and inverse parabolic interpolation (Digital Equipment Corporation, 1982). The magnitude of the surface electric field $\left.\frac{d \psi(x)}{d x}\right|_{x=0}$ can be obtained from the surface charge density $\sigma_{m}$ of the membrane using the charge neutrality condition, Eq. 12. This procedure reduces the solution for the potential $\psi(x)$ to a simple integration of Eq. 63 , as the integrand involves potentials with $y<x$ only. $^{3}$

For the implementation on a computer, it was neccessary to go a different way as the errors introduced by the finite grid size accumulate and cause a violation of the boundary conditions

$$
\psi(x)<0 \quad \text { for all } \quad x \quad \text { and } \quad\left|\psi\left(x_{i}\right)\right|>\left|\psi\left(x_{i+1}\right)\right| \text { for } \quad x_{i}>x_{i+1} .
$$

for large $x$. To enforce these boundary conditions, an iterative method called "shooting" (Davis and Rabinowitz, 1984) was implemented and optimized for our problem. For the GC Model the simplest version of this algorithm shown in Figure 24 was used. As we know, in principle the surface potential to any accuracy, the surface electric field is being varied in order to get a complete solution. Starting from the initial values, calculated as described above, the integration was performed until Eq. 64 was violated. By changing the initial slope of the potential, a solution satisfying the boundary conditions could be found. Numerical tests of the algorithm showed that the deviation of total charge density in the diffuse double layer, $\sigma_{D L}$, obtained by integration and by iteration ${ }^{4}$ from the surface charge density was well below $1 \%$

\footnotetext{
${ }^{3}$ note that for $y=x$ the integrand vanishes

${ }^{4} \sigma_{D L}$ can be obtained from the shooting method ("slope") using the right hand side of Eq. 12
} 


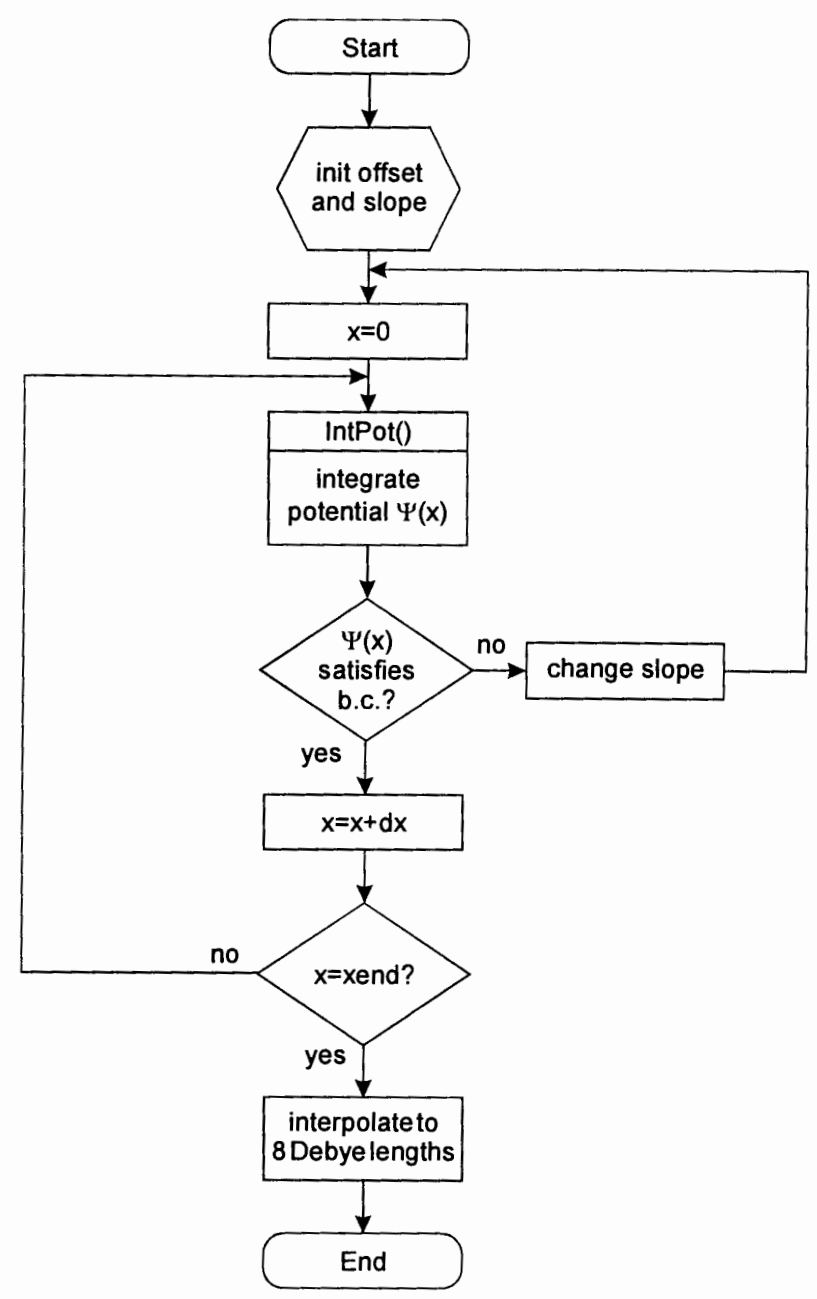

Figure 24: Flowchart for the simple "shooting" algorithm used for solving the GC Model; $\sigma_{m}$ : surface charge density on membrane, $\sigma_{D L}$ : total charge density in diffuse double layer. 
when a grid of 1000 intervals for the first four Debye lengths and interpolation to eight Debye lengths was used. These parameters were kept throughout all calculations except for the adsorption part with the Rod Model where 500 intervals were used instead.

\section{B.3 Numerical Solution of the Rod Model}

The Rod Model, Eqs. 9-11, are very hard to solve as they contain advanced and retarded potentials in $x$. We have two unknowns, the potential and the densities, which each must be determined by iteration. As we deal with integrals over exponentials, a slight change in the potential causes a big change in the densities and vice versa. This leads to an oscillatory behaviour of the solutions. To prevent this from occuring, mixing of iterates (see below) and maximum accuracy for the integration was necessary.

The model equations were solved by extending the procedure for the GC Model. This was necessary, as we have no information on either the surface potential or the surface electric field. As in the case described in Section B.2, the potential is being integrated in an inner loop until the boundary conditions, Eq. 64, are violated. By changing the slope $\left(\left.\frac{d \psi(x)}{d x}\right|_{x=0}\right)$ iteratively, a solution fulfilling the boundary conditions can be found. ${ }^{5}$ After having calculated $\psi(x)$ for a value of $\psi(0)$, the densities for the different charges on RuR, $N_{A C}$ and $N_{B}$ were evaluated on the basis of this potential. It was neccessary to mix the iterates for the density and the potential according to Croxton and McQuarrie (1979) using a mixing parameter $\alpha=0.2$ with

$$
N^{i, n e w}=\alpha N^{i}+(1-\alpha) N^{i-1} \quad \text { and } \quad \psi^{i, n e w}=\alpha \psi^{i}+(1-\alpha) \psi^{i-1}
$$

where $i$ denotes the number of the iterate. The difference between iterates $i-1$ and

\footnotetext{
${ }^{5}$ a solution satisfying the boundary conditions does not exist for all $\psi(0)$; in which case the precision limit of the number representation is reached and the routine quits
} 


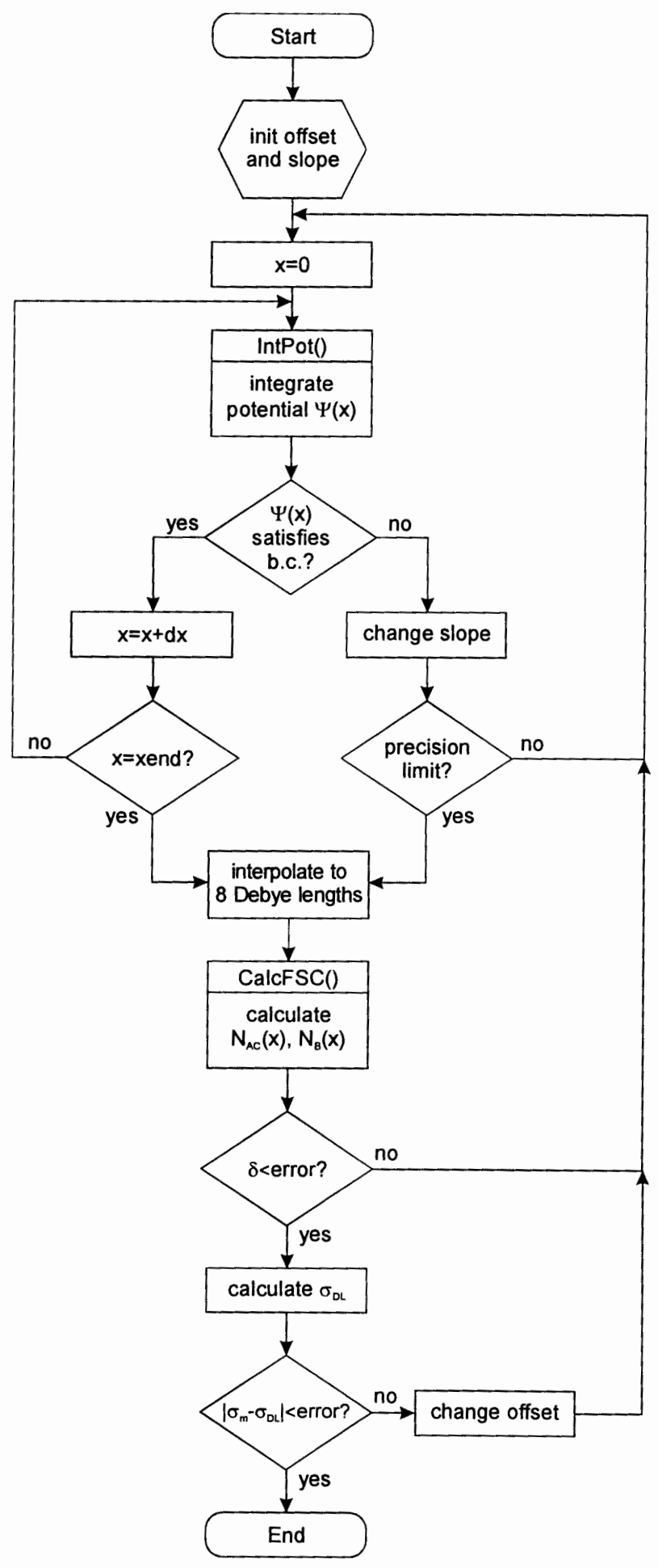

Figure 25: Flowchart for the algorithm used for solving the Rod Model; $\sigma_{m}$ : surface charge density on membrane, $\sigma_{D L}$ : total charge density in diffuse double layer, $\delta$ : relative difference from iteration $i$ to $i+1$, error: a constant determining the maximum error. 
$i$, new for the density was calculated using

$$
\delta=\frac{1}{j_{\max }}\left(\sum_{j}^{j_{\max }}\left(N^{i-1}(j \cdot d x)-N^{i, n e w}(j \cdot d x)\right)^{2}\right)^{\frac{1}{2}}
$$

and compared to the maximum error ${ }^{6}$ allowed.

In an outer loop the offset $(\psi(0))$ is varied until the $\psi(x)$ satisfies the second boundary condition, Eq. 12. The loop for finding the surface potential consisted of the subroutine mentioned in Section B.2 which calculated the root of Eq. 12.

\section{B.4 Numerical Solution of the Maximum Den- sity Model}

The potential for the Maximum Density Model was solved by using the same algorithm as for the Rod Model substituting the densities $N_{A C}$ and $N_{B}$ by $N_{M D}$. This density was obtained by applying to Eq. 13 the root-finding routine mentioned in Section B.2. As no advanced or retarded potentials are involved, the density could be calculated during the potential integration without any additional iteration. The algorithm is outlined in Figure 26.

\section{B.5 Numerical Solution of the Adsorption Model}

The solution for the Adsorption Model is obtained by iterating the surface potential $\psi(0)$ and the final bulk concentration of RuR, $[R u R]_{a q, 0}$. This was done by using the root-finding routine mentioned in Section B.2 twice. For each value of these parameters the Adsorption Model is solved which gives (i) a new value for the surface charge density on the membrane, $\sigma_{m}$, the total charge in the double layer, $\sigma_{D L}$, and (ii) a new bulk concentration for RuR. For a correct solution in order to satisfy all equations, $\sigma_{m}$ must balance $\sigma_{D L}$ and the assumed final bulk concentration

\footnotetext{
${ }^{6} \delta<10^{-3}$ was found to be sufficient
} 


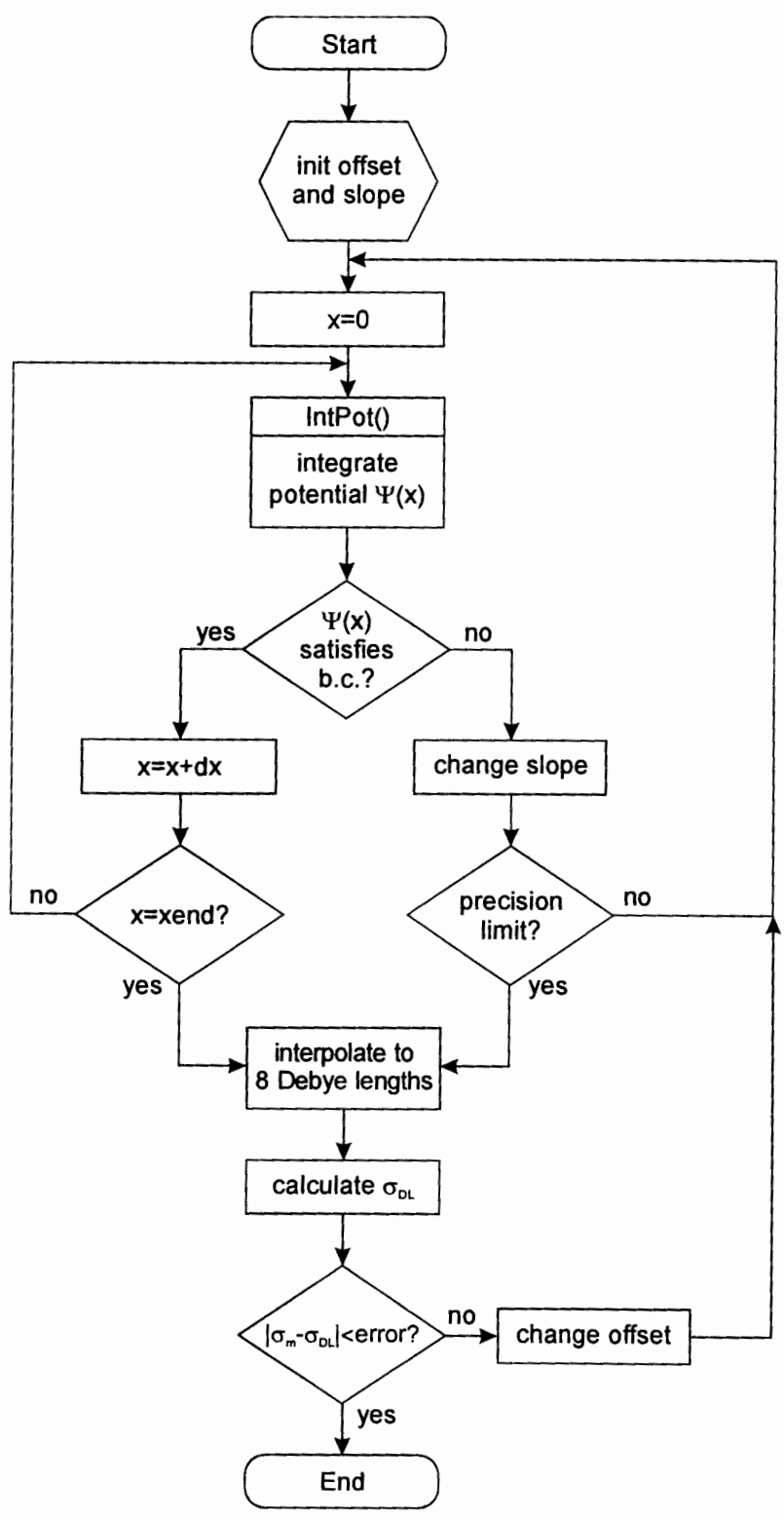

Figure 26: Flowchart for the algorithm used for solving the Maximum Density Model; $\sigma_{m}$ : surface charge density on membrane, $\sigma_{D L}$ : total charge density in diffuse double layer, error: a constant determining the maximum error. 


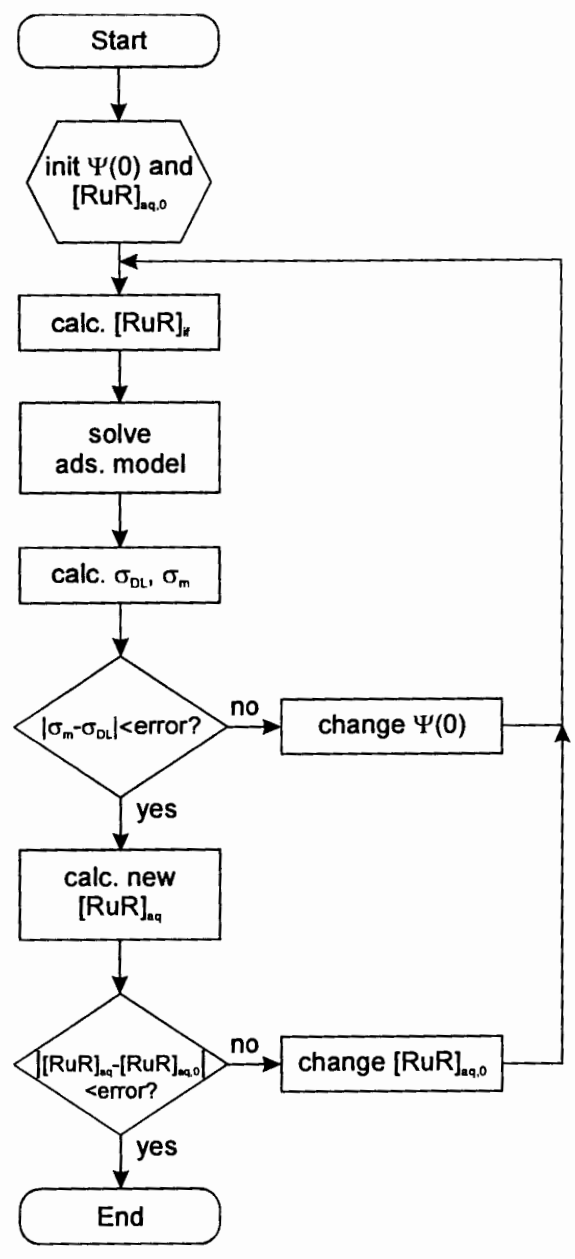

Figure 27: Flowchart for the solution of the Adsorption Model; $\sigma_{m}$ : surface charge density on membrane, $\sigma_{D L}$ : total charge density in diffuse double layer, $[R u R]_{a q, 0}$ : assumed final bulk RuR concentration, $[R u R]_{a q}$ : RuR bulk concentration obtained from Model, $[R u R]_{i f}$ : interfacial RuR concentration, error: a constant determining the maximum error.

$[R u R]_{a q, 0}$ must match the calculated concentration $[R u R]_{a q}$ within the error limits. Solving the Adsorption Model involves the interfacial concentration of RuR which is calculated according to our models. ${ }^{7}$ Figure 27 outlines the numerical procedure.

\footnotetext{
${ }^{7}$ this involves for the Rod and the Maximum Density Model a complete evaluation of $\psi(x)$ as we need the total charge density in diffuse double layer
} 


\section{Appendix C}

\section{Spectrophotometer Control Software}

The main goal for the development of the control software was to achieve an easy management of the spectra and to establish a basis for further data processing of the spectra. This goal was reached by converting the raw data from the spectrophotometer into an ASCII file in which each absorption point corresponds to a wavelength. The file included creation date and time as well as up to 50 comment lines describing the experimental conditions of the obtained data. To automate the data aquisition procedure, data used for a batch of similar measurements can be stored in a "profile". Table 4 lists all parameters stored in a profile and a scan file. The scan additionally includes a [Data] section in which the scan data are listed using the tab character ${ }^{1}$ as delimiter. All files are regular ASCII text files for readability reasons and to facilitate the import of data into other programs for further data processing or display.

The program was written in Visual Basic 3.0 for Windows ${ }^{\circledR}$. It provides a fast and easy way to develop user friendly software using the Windows graphics interface. A button bar gives fast access to the most frequently used functions. The scan data

${ }^{1}$ ASCII (9) 


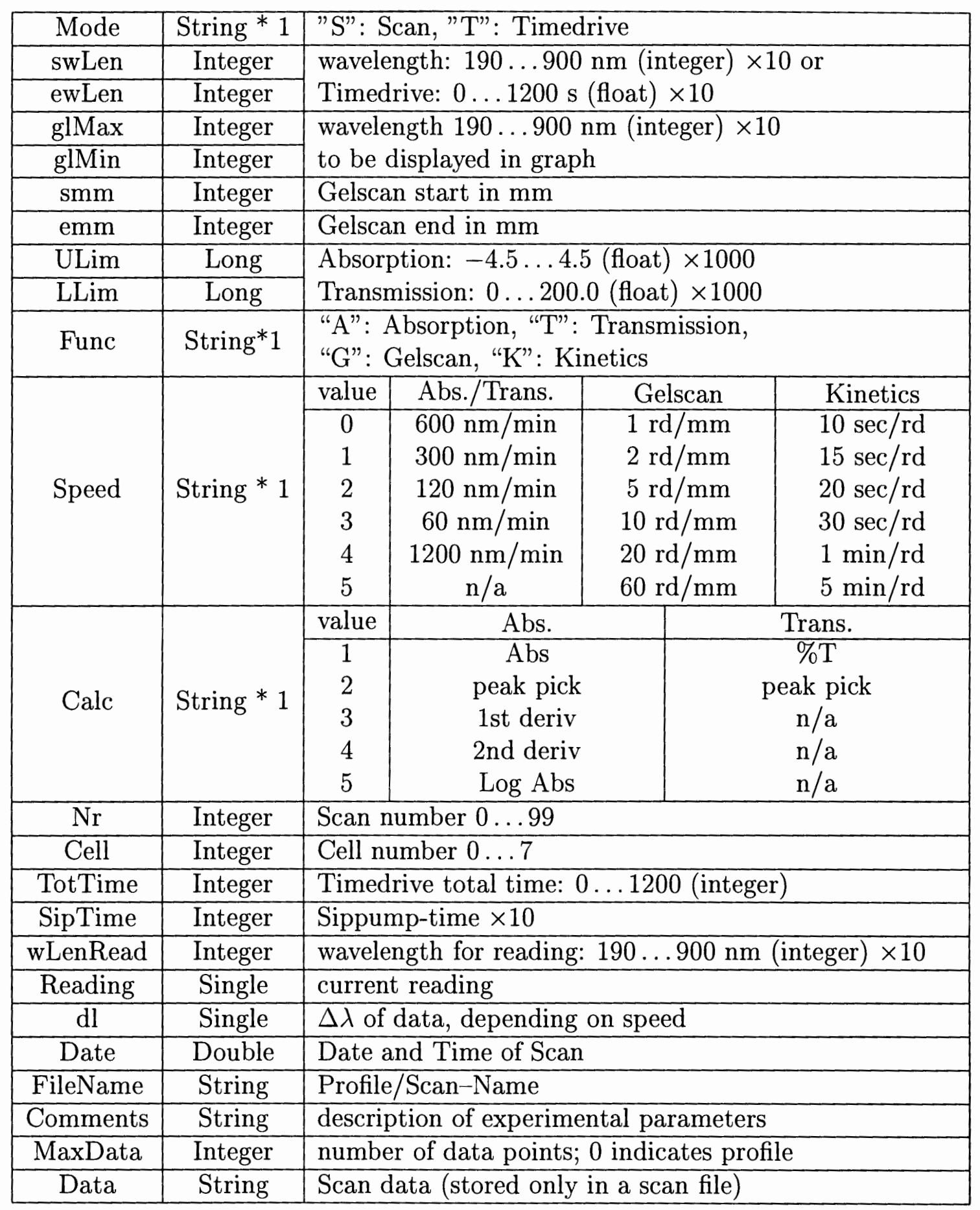

Table 4: List of parameters stored in a profile and a scan file. 


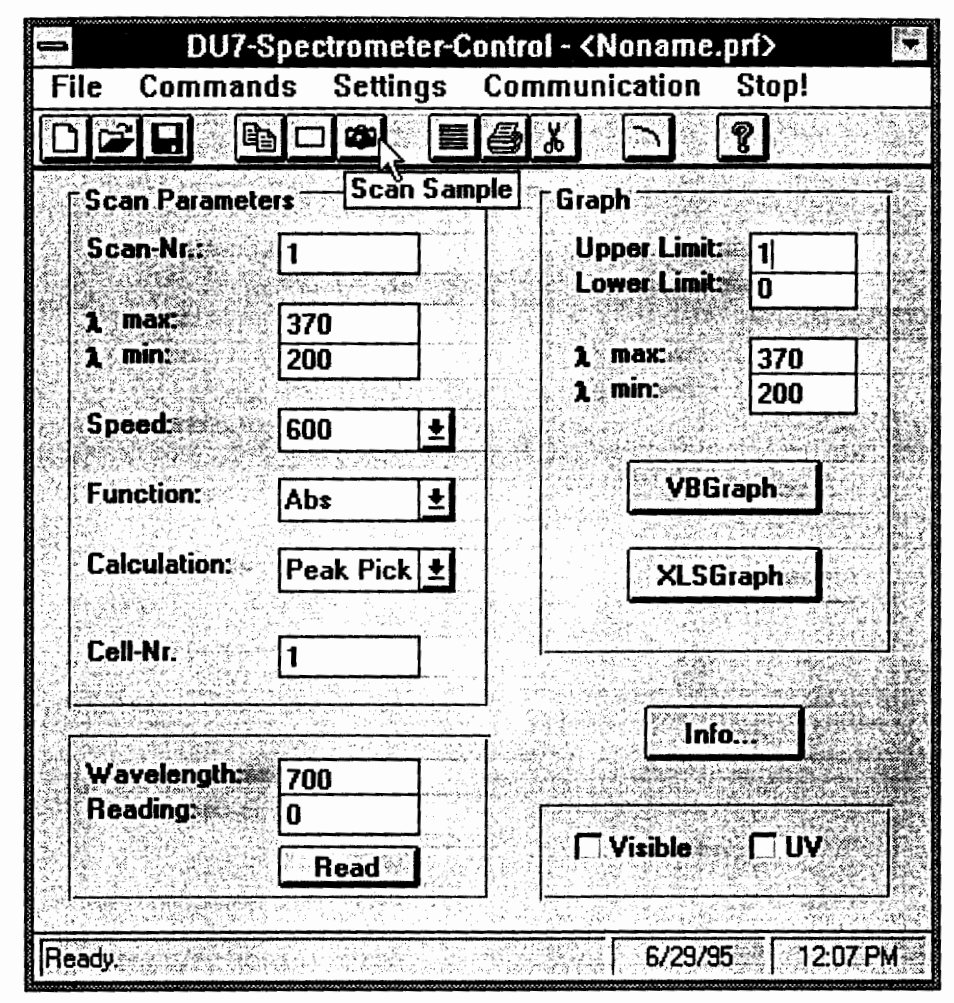

Figure 28: The user interface of the spectrophotometer control software.

is graphically displayed in an extra window which can be interactively positioned and resized. Figure 28 shows the user interface of the program.

The software implements all commands that can be performed on the spectrophotometer via remote control (Beckman, 1984) including the transmission of scan data from and to the spectrophotometer.

Communication between software and spectrophotometer is established by standard serial connection (RS-232). ${ }^{2}$ As the interface for the spectrophotometer does not support hardware handshaking, it was neccessary to slow down the transmission rate by sending character by character to the spectrophotometer rather than a whole

\footnotetext{
${ }^{2}$ using 19200 baud, no parity, 8 data bits and 1 stop bit
} 
command string at once. Characters were received from the spectrophotometer using the polling method ${ }^{3}$.

\footnotetext{
${ }^{3}$ the input port was checked every $100 \mathrm{~ms}$ for incoming data during active communication and every $500 \mathrm{~ms}$ during idle
} 


\section{Bibliography}

Adam, G., Läuger, P., And Stark, G. 1988. Physikalische Chemie und Biophysik. Springer, Berlin; Heidelberg, $2^{\text {nd }}$ edition.

Andersen, O. S., Feldberg, S., and Nakadomarie, H. 1978. Electrostatic interactions among hydrophobic ions in lipid bilayer membranes. Biophys. J. $21: 35-70$.

Aveyard, R. And Haydon, D. A. 1973. An introduction to the principles of surface chemistry. Cambridge University Press.

Barstad, A. W., Peyton, D. H., And Smejtek, P. 1993. AHA- heterodimer of a class-2 uncoupler: Pentachlorophenol. Biochim. Biophys. Acta 1140:262270 .

BECKMAN 1982. DU-7 scanning spectrophotometer service manual. Beckman Instruments, Inc., Fullerton, CA, 92634.

BECKMAN 1984. RS-232 input output accessory. Beckman Instruments, Inc., Irvine, CA, 92713.

Benjamin, I. 1993. Mechanism and dynamics of ion transfer across a liquid-liquid interface. Science 261:1558-1560.

Beschiavili, G. And Seelig, J. 1990. Peptide binding to mixed phosphatidylglycerol/phosphatidylcholine membranes. Biochemistry. 29:52-58.

BhuiYan, L. B. AND Outhwaite, C. W. 1979. Numerical solution of a modified Poisson-Boltzmann equation in electric double layer theory. Chem. Phys. Lett. $66: 321-324$. 
Blochel, A. 1992. Adsorption of halogenated phenolate ions to eggphosphatidylcholine vesicles. Master's thesis, Portland State University.

Brooke, D. N., DobBs, A. J., AND Williams, N. 1986. Octanol:water partition coefficients (P): Measurement, estimation, and interpretation, particularly for chemicals with $P>10^{5}$. Ecotoxicology and Environmental Safety 11:251-260.

Bryant, S. E. And Schultz, T. W. 1994. Toxicological assessment of biotransformation products of pentachlorophenol: Tetrahymena population growth impairment. Arch. Environ. Contam. Toxicol. 26:299-303.

Buff, K., BründL, A., AND BERndT, J. 1982. Differential effects of environmental chemicals in liposomal bilayers: Fluorescence polarization and pesticide-lipid association studies. Biochim. et Biophys. Acta 688:93-100.

Butte, W. 1985. Pentachlorophenol and tetrachlorophenols in wadden sediment and clams Mya arenaria of the Jadebusen after a 14-year period of wastewater discharge containing pentachlorophenol. Environ. Pollut. (G.B.) 9:29.

Carnie, S. ANd MCLaughlin, S. 1983. Large divalent cations and electrostatic potentials adjacent to membranes. Biophys. J. 44:325-332.

Carnie, S. L., Derek, Y. C. C., Mitchell, D. J., and Ninham, B. W. 1981. The structure of electrolytes at charged surfaces: The primitive model. J. Chem. Phys. 15:1472-1478.

Carrondo, M. A. A. F. D. C. T., Griffith, W., Hall, J., and Skapski, A. 1980. X-ray structure of $\left[\mathrm{Ru}_{3} \mathrm{O}_{2}\left(\mathrm{NH}_{3}\right) \mathrm{I}_{4}\right]^{+6}$. Biochim. Biophys. Acta 627:332334.

Cazabat, A. M. And Langevin, D. 1980. Light scattering by water in oil microemulsions., pp. 139-172. In V. Degiorgio (ed.), Light scattering in liquids and macromolecular solutions. Plenum Press, New York, NY, 10011.

Chapman, D. L. 1913. A contribution to the theory of electrocapillarity. Philos. Mag. 25:475-481.

Cireldi, D. P. 1978. Pentachlorophenol position document 1. Fed. Reg. 43:4844648477. 
Crosby, D. G. 1981. Environmental chemistry of pentachlorophenol. Pure Appl. Chem. 53:1051-1080.

Croxton, S. L. ANd McQuarrie, D. A. 1979. Numerical solution of the BornGreen-Yvon equation for the restricted primitive model of ionic solutions. $J$. Phys. Chem. 83:1840-1843.

Davis, J. P. And Rabinowitz, P. 1984. Methods of numerical integration. Academic Press, Orlando, FL 32887, $2^{\text {nd }}$ edition.

De Bruijn, J. AND Hermens, J. 1990. Relationships between octanol/water partition coefficients and total molecular surface area and total molecular volume of hydrophobic organic chemicals. Quant. Struct.-Act. Relat. 9:11-21.

Digital Equipment CoRporation 1982. Scientific subroutines SSP V1.3.

Dougherty, R. C. 1978. Human exposure to pentachlorophenol., p. 351. In K. R. Rao (ed.), Pentachlorophenol: Chemistry, pharmacology, and environmental toxicology. Plenum Press, New York.

Duniec, J. T. And Thorne, S. W. 1983. Electrostatic potentials in membrane systems. Bull. Math. Biol. 45:69-90.

Duxbury, C. L. And Thompson, J. E. 1987. Pentachlorophenol alters the molecular organization of membranes in mammalian cells. Arch. Environ. Contam. Toxicol. 16:367--373.

Flewelling, R. F. And Hubbell, W. L. 1986. The membrane dipole potential in a total membrane potential model. Biophys. J. 49:541-552.

Geyer, H. J., Scheunert, I., And Korte, F. 1987. Distribution and bioconcentration potential of the environmental chemical pentachlorophenol in different tissues of humans. Chemosphere 16:887-899.

Gouy, M. 1910. Sur la constitution de la charge électrique à la surface d'un électrolyte. J. Phys. (Paris) 9:457-468.

Grahame, D. C. 1947. The electical double layer and the theory of electrocapillarity. Chem. Rev. 41:441.

Graney, R. L. And Giesy, JR., J. P. 1987. The effect of short-term exposure to 
pentachlorophenol and osmotic stress on the free amino acid pool of freshwater Amphipod Gammarus pseudolinaesu Bousfield. Arch. Environ. Contam. Toxicol. 16:167-176.

Harned, H. S. AND OWEn, B. B. 1958. The physical chemistry of electrolytic solutions. American Chemical Society, Washington, DC.

Hattemer-Frey, H. A. And Travis, C. C. 1989. Pentachlorophenol: Environmental partitioning and human exposure. Arch. Environ. Contam. Toxicol. 18:482-489.

Henderson, D., Blum, L., And Smith, W. R. 1979. Application of the hypernetted chain approximation to the electric double layer at a charged planar interface. Chem. Phys. Lett. 63:381-383.

Jayaweera, R., Petersen, R., and Smejtek, P. 1982. Induced hydrogen ion transport in lipid membranes as origin of toxic effect of pentachlorophenol in an alga. Pesticide Biochemistry and Physiology 18:197-204.

KAISER, K. L. E. AND VAldmanis, I. 1982. Apparent octanol/water partition coefficients of pentachlorophenol as a function of $p H$. Can. J. Chem. 60:21042106.

Kamlet, M. J., Doherty, R. M., Carr, P. W., Mackay, D., Abraham, M. H., AND TAFT, R. W. 1988. Linear solvation energy relationships. 44. Parameter estimation rules that allow accurate prediction of octanol/water partition coefficients and other solubility and toxicity properties of polychlorinated biphenyls and polycyclic aromatic hydrocarbons. Environ. Sci. Technol. 22:503509.

Kaufman, D. D. 1978. Degradation of pentachlorophenol in soil, and by soil microorganisms, p. 27. In K. R. Rao (ed.), Pentachlorophenol: Chemistry, pharmacology, and environmental toxicology. Plenum Press, New York.

Kerler, F. AND SchönherR, J. 1988. Accumulation of lipophilic chemicals in plant cuticles: Prediction from octanol/water partition coefficients. Arch. Environ. Contam. Toxicol. 17:1-6. 
KJEllander, R. AND MARČElJA, S. 1985. Inhomogeneous coulomb fluids with image interactions between planar surfaces. I. J. Chem. Phys. 82:2122-2135.

KJELLANDER, R. AND MARČELJA, S. 1988a. Inhomogeneous coulomb fluids with image interactions between planar surfaces. II. On the anisotropic hypernetted chain approximation. J. Chem. Phys. 88:7129-7137.

KJELlander, R. AND MARČELJA, S. 1988b. Inhomogeneous coulomb fluids with image interactions between planar surfaces. III. Distribution functions. J. Chem. Phys. 88:7138-7146.

KLEIN, R. 1994. Statistische Mechanik. Universität Konstanz, Konstanz, Germany. Script zur Vorlesung.

Klopman, G., Krishnan, N., And Schochet, M. 1985. Simple method of computing partition coefficient. J. Comp. Chem. 6:28-38.

KuCHINKA, E. AND SeELig, J. 1989. Interaction of melittin with phosphatidylcholine membranes. Biochemistry. 28:4216-4221.

Lagas, P. 1988. Sorption of chlorophenols in the soil. Chemosphere 17:205-216.

Langner, M., Cafiso, D., Marčelja, S., and Mclaughlin, S. 1990. Electrostatics of phosphoinositide bilayer membranes. Biophys. J. 57:335-349.

Leo, A., Hansch, C., And Elkins, D. 1971. Partition coefficients and their uses. Chem. Rev. 6:525-616.

Linder, M. E. And Gilman, A. G. 1992. G proteins. Scientific American 267:56-65 .

MACKAY, D. 1982. Correlation of bioconcentration factors. Environ. Sci. Technol. 16:274-278.

McIntosh, T. J. And Simon, S. A. 1986. Hydration force and bilayer deformation: A reevaluation. Biochemistry 25:4058-4066.

MCLAUGHLin, S. 1977. Electrostatic potentials at membrane-solution interfaces. Curr. Top. Membr. Transp. 9:71-144.

Mikesell, M. D. And BoYd, S. D. 1986. Complete reductive dechlorination and mineralization of pentachlorophenol by anaerobic microorganisms. Appl. 
Environ. Microbiol. 52:861-865.

MiLleR, M. M. AND WASIK, S. P. 1985. Relationships between octanol-water partition coefficient and aqueous solubility. Environ. Sci. Technol. 19:522-529. Montich, G., Scarlata, S., Mclaughlin, S., Lehrmann, R., and Seelig, J. 1993. Thermodynamic characterization of the association of small basic peptides with membranes containing acidic lipids. Biochim. Biophys. Acta 1146:1724.

Mosior, M. AND MCLaughlin, S. 1992. Electrostatics and dimensionality can produce apparent cooperativity when protein kinase $\mathrm{C}$ and its substrates bind to acidic lipids in membranes, pp. 157-180. In R. Epand and D. Lester (eds.), Protein kinease C: Current concepts and future perspectives, chapter 9. Ellis Horwood, Chichester, England.

Murthy, N. B. K., Kaufman, D. D., and Fries, G. F. 1979. Degradation of pentachlorophenol (PCP) in aerobic and anaerobic soil. J. Environ. Qual. 14:337-340.

NELson, A. P. ANd MCQUarRie, D. A. 1975. The effect of discrete charges on the electrical properties of a membrane. J. theor. Biol. 55:13-27.

OECD 1981. Partition coefficients. In Guidelines for testing chemicals, volume 107, Paris.

OuthwaIte, C. W. 1974. Higher-order closures and potential problems in diffuse double layer and strong electrolyte theory. Mol. Phys. 27:561-575.

Paasivirta, J., Tenhola, H., Palm, H., and Lammi, R. 1992. Free and bound chlorophenols in Kraft pulp bleaching effluents. Chemosphere 9:1253-1258.

Peitzsch, R. M., Eisenberg, M., Sharp, K. A., and McLaughlin, S. 1995. Calculations of the electrostatic potential adjacent to model phospholipid bilayers. Biophys. J. 68:729-738.

Rogers, I. H., MacDonald, J. S., ANd SAdar, M. 1992. Uptake of selected organochlorine contaminants in fishes resident in the Fraser River Estuary, Vancouver, British Columbia. Water Poll. Res. J. Canada 27:733--749. 
SABLIJć, A. 1991. Chemical topology and ecotoxicology. The Science of the Total Environment 109/110:197-220.

Schellenberg, K., Leuenberger, C., and Schwarzenbach, R. P. 1984. Sorption of chlorinated phenols by natural sediments and aquifier materials. Environ. Sci. Technol. 18:652-657.

SCHMIDT, P. O. 1995. SEM-imaging of artificial liposomes. unpublished.

Schoch, P. And Sargent, D. F. 1980. Quantitative analysis of the binding of melittin to planar lipid bilayers allowing for the discrete charge effect. Biochim. Biophys. Acta 602:234-247.

Schwarz, G. And Beschiaschvili, G. 1989. Thermodynamic and kinetic studies on the association of melittin with a phospholipid bilayer. Biochim. Biophys. Acta. 979:82-90.

Singer, S. J. AND NiColson, G. L. 1972. The fluid mosaic model of the structure of cell membranes. Science 175:720-731.

SMejtek, P. 1987. The physicochemical basis of the membrane toxicity of pentachlorophenol: An overview. Journal of Membrane Science 33:249-268.

Smejtek, P., Barstad, A. W., ANd Wang, S. 1989. Pentachlorophenol-induced change of $\zeta$-potential and gel-to-fluid transition temperature in model lecithin membranes. Chem.-Biol. Interactions 71:37-61.

SMEJTEK, P. AND WANG, S. 1993. Distribution of hydrophobic ionizable xenobiotics between water and lipid membranes: Pentachlorophenol and pentachlorophenate. Arch. Environ. Contam. Toxicol. 25:394-404.

Smit, B., Hilbers, P. A. J., Esselink, K., Rupert, L. A. M., van Os, N. M., AND Schlijper, A. G. 1990. Computer simulations of a water/oil interface in the presence of micelles. Nature 348:624-625.

StAnKowski, S. 1991. Surface charging by large multivalent molecules. Biophys. J. 60:341-351. 
Stankowski, S. AND Schwarz, G. 1990. Electrostatics of a peptide at a membrane/water interface. The $p H$ dependence of melittin association with lipid vesicles. Biochim. Biophys. Acta. 1025:164-172.

Stern, O. 1924. Zur Theorie der elektrolytischen Doppelschicht. A. Elektrochem. $30: 508$.

Stryer, L. 1981. Biochemistry. W. H. Freeman and Company, San Francisco.

TERADA, H. 1990. Uncouplers of oxidative phosphorylation. Environmental Health Perspectives 87:213-218.

Torrie, G. M. And Valleau, J. P. 1979. A Monte Carlo study of an electrical double layer. Chem. Phys. Lett. 65:343-346.

Torrie, G. M. and Valleau, J. P. 1980. Electrical double layers. I. Monte Carlo study of a uniformly charged surface. J. Chem. Phys. 73:5807-5816.

Torrie, G. M., Valleau, J. P., and Patey, G. N. 1982. Electrical double layers. II. Monte Carlo and HNC studies of image effects. J. Chem. Phys. 76:4615-4622.

Tsien, R. Y. And Hladky, S. B. 1982. Ion repulsion within membranes. Biophys. J. 39:49-56.

van Gestel, C. A. M., Otermann, K., and Canton, J. H. 1985. Relation between water solubility, octanol/water partition coefficients, and bioconcentration of organic chemicals in fish: A review. Regulatory Toxicology and Pharmacology 5:422-431.

VAn Megen, W. And Snook, I. 1980. The grand canonical ensemble Monte Carlo method applied to the electrical double layer. J. Chem. Phys. 73:4656-4662.

Veith, G. D. And Mekenyan, O. G. 1993. A QSAR approach for estimating the aquatic toxicity of soft electrophiles. Quant. Struct.-Act. Relat. 12:349-356.

VölKer, D. AND Smejtek, P. 1995. Adsorption of Ruthenium Red to phospholipid membranes. Biophys. J. (submitted) .

Wasik, S. P., Tewari, Y. B., Miller, M. M., and Martire, D. E. 1981. Octanol/water partition coefficients and aqueous solubility of organic compounds. 
U.S. Department of Commerce/National Bureau of Standards, NBSIR 81:2406. Weber, JR., W. J., Chin, Y.-P., ANd Rice, C. P. 1986. Determination of partitioning coefficients and aqueous solubilities by reverse phase chromatography I, II. Wat. Res. 20:1433-1450.

WeINBACH, J. C. 1956. Biochemical basis for the toxicity of pentachlorophenol. Science 124:940.

Westall, J. C., Leuenberger, C., and Schwarzenbach, R. 1985. Influence of $p H$ and ionic strength on the aqueous-nonaqueous distribution of chlorinated phenols. Environ. Sci. Technol. 19:193-198.

Wild, S. R. AND JonEs, K. C. 1992. Pentachlorophenol in the U.K. environment, II. Chemosphere 24:847-855.

Winiski, A. P., Mclaughlin, A. C., McDaniel, R. V., Eisenberg, M., AND MCLaughlin, S. 1986. An experimental test of the discreteness-of-charge effect in positive and negative lipid bilayers. Biochemistry 25:8206-8214.

Woodburn, K. B., Doucette, W. J., And Andren, A. W. 1984. Generator column determination of octanol/water partition coefficients for selected polychlorinated biphenyl congeners. Environ. Sci. Technol. 18:457-459. 